\title{
Symmetric Nonbarotropic Flows with Large Data and Forces
}

\author{
DAVID HoFF \& Helge KRISTIAN JENSSEN
}

\author{
Communicated by Y. BRENIER
}

\begin{abstract}
We prove the global existence of weak solutions of the Navier-Stokes equations of compressible, nonbarotropic flow in three space dimensions with initial data and external forces which are large, discontinuous, and spherically or cylindrically symmetric. The analysis allows for the possibility that a vacuum state emerges at the origin or axis of symmetry, and the equations hold in the sense of distributions in the set where the density is positive. In addition, the mass and momentum equations hold weakly in the entire space-time domain, but with a nonstandard interpretation of the viscosity terms as distributions. Solutions are obtained as limits of solutions in annular regions between two balls or cylinders, and the analysis allows for the possibility that energy is absorbed into the origin or axis, and is lost in the limit as the inner radius goes to zero.
\end{abstract}

\section{Introduction}

We prove the global existence of weak solutions of the Navier-Stokes equations of compressible, nonbarotropic flow in three space dimensions with initial data and external forces which are large, discontinuous, and spherically or cylindrically symmetric. In the latter case the spatial region is an infinite cylinder, and in the former a ball. In both cases the velocity and temperature gradient vanish at the boundary. The analysis allows for the possibility that a vacuum state emerges at the origin or axis of symmetry, and the equations hold in the sense of distributions in the set where the density is positive. In addition, the mass and momentum equations hold weakly in the entire space-time domain, but with a nonstandard interpretation of the viscosity terms as distributions. The energy equation is shown only to hold weakly on the support of the density. Solutions are obtained as limits of solutions in annular regions between two balls or cylinders, and the analysis allows for the possibility that energy is absorbed into the origin or axis, and is lost in the limit as the inner radius goes to zero. 
The Navier-Stokes equations of compressible, nonbarotropic flow express the conservation of mass and the balance of momentum and energy as follows:

$$
\begin{aligned}
\rho_{t}+\operatorname{div}(\rho \mathbf{U})= & 0 \\
\left(\rho \mathbf{U}^{i}\right)_{t}+\operatorname{div}\left(\rho \mathbf{U}^{i} \mathbf{U}\right)+P(\rho, \theta)_{x_{i}}= & \mu \Delta \mathbf{U}^{i}+(\lambda+\mu) \operatorname{div} \mathbf{U}_{x_{i}}+\rho \mathbf{F}^{i} \\
(\rho E)_{t}+\operatorname{div}((\rho E+P(\rho, \theta)) \mathbf{U})= & \Delta\left(\kappa \theta+\frac{1}{2} \mu|\mathbf{U}|^{2}\right)+\mu \operatorname{div}((\nabla \mathbf{U}) \mathbf{U}) \\
& +\lambda \operatorname{div}((\operatorname{div} \mathbf{U}) \mathbf{U})+\rho \mathbf{U} \cdot \mathbf{F} .
\end{aligned}
$$

Here $x \in \mathbb{R}^{3}$ is the spatial coordinate, $t>0$ is time, $\rho, \mathbf{U}=\left(\mathbf{U}^{1}, \mathbf{U}^{2}, \mathbf{U}^{3}\right)$, $\theta, E=\frac{1}{2}|\mathbf{U}|^{2}+\theta$, and $P=K \rho \theta$ are respectively the density, velocity, temperature, energy density, and pressure of an ideal fluid (with unit specific heat); $\mu$ and $\lambda$ are positive viscosity constants, and $\kappa$ is a positive heat-conduction coefficient; $\mathbf{F}=\left(\mathbf{F}^{1}, \mathbf{F}^{2}, \mathbf{F}^{3}\right)$ is the external force, and $\nabla \mathbf{U}$ denotes the Jacobian of the velocity vector with respect to the space variables.

We pose the system (1)-(3) in either a ball $\Omega$ of radius $b$ centered at the origin in $\mathbf{R}^{3}$, or in an infinite cylinder of radius $b$ parallel to and centered along the $x_{3}$ axis in $\mathbf{R}^{3}$, also denoted by $\Omega$. In both cases we impose the boundary conditions

$$
\mathbf{U}=0, \theta_{r}=0 \text { on } \partial \Omega,
$$

and we denote the initial conditions by

$$
\left.(\rho, \mathbf{U}, \theta)\right|_{t=0}=\left(\rho_{0}, \mathbf{U}_{0}, \theta_{0}\right)
$$

For the symmetric cases under consideration here, (1)-(3) take the form

$$
\begin{aligned}
\rho_{t}+(\rho u)_{\xi} & =0 \\
(\rho u)_{t}+\left(\rho u^{2}\right)_{\xi}-\frac{\rho v^{2}}{r}+P(\rho, \theta)_{r}-v u_{\xi r} & =\rho f_{1} \\
(\rho v)_{t}+(\rho u v)_{\xi}+\frac{\rho u v}{r}-\mu v_{\xi r} & =\rho f_{2} \\
(\rho w)_{t}+(\rho u w)_{\xi}-\mu w_{r \xi} & =\rho f_{3} \\
(\rho \theta)_{t}+(\rho u \theta)_{\xi}-\kappa \theta_{r \xi}+P(\rho, \theta) u_{\xi}-Q & =0
\end{aligned}
$$

where

$$
v:=\lambda+2 \mu, \quad \frac{\partial}{\partial \xi}=\frac{\partial}{\partial r}+\frac{m}{r},
$$

$m=1$ or 2 , and $Q$ is the homogeneous quadratic

$$
Q=v\left(u_{\xi}\right)^{2}+\mu\left(v_{\xi}\right)^{2}+\mu\left(w_{r}\right)^{2}-\frac{2 \mu m}{r}\left(v^{2}\right)_{r}-\frac{2 \mu m}{r^{m}}\left(r^{m-1} u^{2}\right)_{r} .
$$

Although not immediately apparent, it is straightforward to check that $Q$ is positive definite in $u \xi, u / r, v_{r}-m v / r$, and $w_{r}$. In the spherically symmetric case, $m=$ $2, r(x)=|x|$,

$$
\mathbf{U}(x, t)=u(r, t) \frac{x}{r}, \quad \mathbf{F}(x, t)=f_{1}(r, t) \frac{x}{r},
$$


and $v=w \equiv 0$. In the cylindrically symmetric case, $m=1, r(x)=\sqrt{x_{1}^{2}+x_{2}^{2}}$, and

$$
\mathbf{U}(x, t)=u(r, t) \frac{\left(x_{1}, x_{2}, 0\right)}{r}+v(r, t) \frac{\left(-x_{2}, x_{1}, 0\right)}{r}+w(r, t)(0,0,1),
$$

F being constructed from $\left(f_{1}, f_{2}, f_{3}\right)$ in a similar way. Thus $\mathbf{F}^{i}$ are the components of the vector $\mathbf{F}$ in Cartesian coordinates, and $\left(f_{1}, f_{2}, f_{3}\right)$ the components in spherical or cylindrical coordinates, with $f_{2}=f_{3}=0$ in the spherical case. Similarly, $\mathbf{U}^{i}$ are the components of the vector $\mathbf{U}$ in Cartesian coordinates, and $(u, v, w)$ the components in spherical or cylindrical coordinates, with $v=w=0$ in the spherical case. Abusing notation slightly, we write $\rho(x, t)=\rho(r(x), t)$ and $\theta(x, t)=\theta(r(x), t)$.

We now give precise statements of our assumptions and results. The external force $\mathbf{F}$ is assumed to satisfy

$$
\mathbf{F} \in L^{1}\left([0, T] ; L^{\infty}(\Omega)\right) \cap L^{\infty}\left([0, T] ; L^{q}(\Omega)\right)
$$

for each $T>0$ and for some $q>2$, which will be fixed throughout. The initial data $\left(\rho_{0}, \mathbf{U}_{0}, \theta_{0}\right)$ is assumed to satisfy

$$
\begin{aligned}
& C_{0}^{-1} \leqq \rho_{0} \leqq C_{0}, \quad C_{0}^{-1} \leqq \theta_{0} \text { a.e., } \\
& \int_{\Omega} \rho_{0} S\left(\rho_{0}, \mathbf{U}_{0}, \theta_{0}\right) d x \leqq C_{0}, \\
& \int_{0}^{b} \rho_{0}(r)\left[\left|r v_{0}(r)\right|^{q}+\left|w_{0}(r)\right|^{q}\right] r^{m} d r \leqq C_{0},
\end{aligned}
$$

for a constant $C_{0}$. Here $S$ is the entropy density

$$
S(\rho, \mathbf{U}, \theta)=K \Psi\left(\rho^{-1}\right)+\Psi(\theta)+\frac{1}{2}|\mathbf{U}|^{2},
$$

where $\Psi(s)=s-\log s-1$. Observe that there are no smallness or regularity conditions imposed on $\mathbf{F}$ or on $\left(\rho_{0}, \mathbf{U}_{0}, \theta_{0}\right)$.

We obtain a global weak solution as the limit as $j \rightarrow \infty$ of approximate smooth solutions $\left(\rho^{j}, \mathbf{U}^{j}, \theta^{j}\right)$ in annular regions $\Omega_{j}=\left\{x \mid a_{j}<r(x)<b\right\}$, where $a_{j}$ is a sequence of positive inner radii tending to zero, with mollified initial data $\left(\rho_{0}^{j}, \mathbf{U}_{0}^{j}, \theta_{0}^{j}\right)$. In describing the limits of these approximate solutions we fix an increasing $C^{1}$ function $\chi:[0, \infty) \rightarrow[0,1]$ with $\chi \equiv 0$ on $[0,1]$ and $\chi \equiv 1$ on $[2, \infty)$, and for $R>0$ we define $\chi^{R}(r)=\chi(r / R)$.

The following theorem contains the main results of this paper:

Theorem 1.1. Assume that the system parameters $\lambda, \mu$, and $\kappa$ are positive, that $P(\rho, \theta)=K \rho \theta$, where $K$ is a constant, and that the external force $\mathbf{F}$ and the initial data $\left(\rho_{0}, \mathbf{U}_{0}, \theta_{0}\right)$ satisfy the conditions (12)-(14). Then the initial-boundary problem (1)-(5) has a global weak solution $(\rho, \mathbf{U}, \theta)$ for which the support of $\rho$ is bounded on the left by a curve $\underline{r}(t)$, satisfying the following: 
(a) The function $\underline{r}:[0, \infty) \rightarrow[0, \infty)$ is a semicontinuous curve, so that, if $\mathcal{F}$ is the set

$$
\mathcal{F}:=\{(x, t): t \geqq 0 \text { and } \underline{r}(t)<r(x) \leqq b\},
$$

then $\mathcal{F} \cap\{t>0\} \cap\{r<b\}$ is open.

(b) The density $\rho \in L_{\mathrm{loc}}^{\infty}(\mathcal{F})$, $\mathbf{U}$ and $\theta$ are locally Hölder continuous in $\mathcal{F} \cap\{t>0\}$, and the Navier-Stokes equations (1)-(3) hold in $\mathcal{D}^{\prime}(\mathcal{F} \cap\{t>0\} \cap\{r<b\})$.

(c) The density $\rho \in C\left([0, \infty) ; W^{1, \infty}(\Omega)^{*}\right)$ in the spherically symmetric case, and is in $C\left([0, \infty) ; W_{L}^{1, \infty}(\Omega)^{*}\right)$ in the cylindrically symmetric case, where functions in $W_{L}^{1, \infty}$ vanish for $\left|x_{3}\right|>L$, with $\rho(\cdot, 0)=\rho_{0}$. Also, $\rho(\cdot, t) \equiv 0$ in $\Omega \backslash \overline{\mathcal{F}}$, and if $\rho \mathbf{U}$ is taken to be zero in $\Omega \backslash \overline{\mathcal{F}}$, then the weak form of the mass equation (1) holds for test functions $\psi \in C^{1}\left(\bar{\Omega} \times\left[t_{1}, t_{2}\right]\right)$ which are assumed to vanish for $\left|x_{3}\right| \geqq L$ for some $L>0$ in the cylindrically symmetric case:

$$
\left.\int_{\Omega} \rho \psi d x\right|_{t_{1}} ^{t_{2}}=\int_{t_{1}}^{t_{2}} \int_{\Omega}\left(\rho \psi_{t}+\rho \mathbf{U} \cdot \nabla \psi\right) d x d t .
$$

(d) For $t_{1}<t_{2}$ fixed there are distributions $\mathfrak{U}_{i} \in C^{2}\left(\bar{\Omega} \times\left[t_{1}, t_{2}\right]\right)^{*}$ in the spherically symmetric case, and in $C_{L}^{2}\left(\bar{\Omega} \times\left[t_{1}, t_{2}\right]\right)^{*}$ in the cylindrically symmetric case, where functions in $C_{L}^{2}$ vanish for $\left|x_{3}\right|>L$, such that the weak form of the momentum equation (2) holds in the following sense: if $\psi \in C^{2}\left(\bar{\Omega} \times\left[t_{1}, t_{2}\right]\right)$ vanishes on $\partial \Omega$, and vanishes for $\left|x_{3}\right|>L$ for some $L$ in the cylindrically symmetric case, then

$$
\begin{gathered}
\left.\int_{\Omega} \rho \mathbf{U}^{i} \psi d x\right|_{t_{1}} ^{t_{2}}-\int_{t_{1}}^{t_{2}} \int_{\Omega}\left(\rho \mathbf{U}^{i} \psi_{t}+\rho \mathbf{U}^{i} \mathbf{U} \cdot \nabla \psi+P \psi_{x_{i}}\right) d x d t \\
=\int_{t_{1}}^{t_{2}} \int_{\Omega} \rho \mathbf{F}^{i} \psi d x d t+\mathfrak{U}_{i}(\psi) .
\end{gathered}
$$

The distribution $\mathfrak{U}_{i}$ is given by

$$
\mathfrak{U}_{i}(\psi)=\lim _{R \rightarrow 0} \lim _{j \rightarrow \infty} \int_{t_{1}}^{t_{2}} \int_{\Omega}\left[(\lambda+\mu) \mathbf{U}^{j} \cdot \nabla \psi_{x_{i}}^{R}+\mu\left(\mathbf{U}^{j}\right)^{i} \Delta \psi^{R}\right] d x d t
$$

where

$$
\psi^{R}(x, t):=\chi^{R}(|x|) \psi(x, t)
$$

for $i=1,2,3$ in the spherically symmetric case and for $i=1,2$ in the cylindrically symmetric case, and

$$
\psi^{R}(x, t):=\psi\left(0, x_{3}, t\right)+\chi^{R}(|x|)\left[\psi(x, t)-\psi\left(0, x_{3}, t\right)\right]
$$

for $i=3$ in the cylindrically symmetric case. 
(e) The gradients $\nabla \mathbf{U}, \nabla \theta \in L_{\text {loc }}^{1}(\mathcal{F})$, and the weak form of the energy equation (3) holds for test functions $\psi \in C^{2}\left(\bar{\Omega} \times\left[t_{1}, t_{2}\right]\right)$ for which there is an $\eta>0$ such that $\operatorname{supp} \psi(\cdot, t) \subset\{x: \underline{r}(t)+\eta \leqq r(x)\}$ for each $t \in\left[t_{1}, t_{2}\right]$ (and such that $\psi=0$ for $\left|x_{3}\right| \geqq L$, for some $L>0$ in the cylindrically symmetric case):

$$
\begin{aligned}
\left.\int_{\Omega} \rho E \psi d x\right|_{t_{1}} ^{t_{2}}-\int_{t_{1}}^{t_{2}} \int_{\Omega}\left(\rho E \psi_{t}+(\rho E+P) \mathbf{U} \cdot \nabla \psi\right) d x d t \\
=\int_{t_{1}}^{t_{2}} \int_{\Omega} \rho \mathbf{F} \cdot \mathbf{U} \psi d x d t \\
\quad-\int_{t_{1}}^{t_{2}} \int_{\Omega}\left(\kappa \nabla \theta+\frac{1}{2} \mu \nabla|\mathbf{U}|^{2}+\mu(\operatorname{div} \mathbf{U}) \mathbf{U}+\lambda(\nabla \mathbf{U}) \mathbf{U}\right) \cdot \nabla \psi d x d t
\end{aligned}
$$

(f) The total energy, minus the mechanical work done by the external force, is weakly nonincreasing in time. That is, if

$$
\mathcal{E}(t):=\int_{\Omega} \rho(x, t)\left[\theta(x, t)+\frac{1}{2}|\mathbf{U}|^{2}\right] d x
$$

in the spherically symmetric case, then

$$
\mathcal{E}(T)=\mathcal{E}(0)+\int_{0}^{T} \int_{\Omega} \rho \mathbf{U} \cdot \mathbf{F} d x d t-\lim _{R \rightarrow 0} \lim _{j \rightarrow \infty} \int_{a_{j} \leqq r(x) \leqq R}\left(\rho^{j} E^{j}\right)(x, T) d x
$$

as a function of $T$ in $\mathcal{D}^{\prime}(0, \infty)$, where $E^{j}=\frac{1}{2}\left|\mathbf{U}^{j}\right|^{2}+\theta^{j}$. A similar result holds in the cylindrically symmetric case, but with all spatial integrations over the set $\left\{x: r(x)<b\right.$ and $\left.x_{3}=0\right\}$.

We now give a brief description of the analysis and we make a number of comments concerning the interpretation of the above results. As indicated earlier, the solution $(\rho, \mathbf{U}, \theta)$ is obtained as the limit as $j \rightarrow \infty$ of solutions $\left(\rho^{j}, \mathbf{U}^{j}, \theta^{j}\right)$ in the annular region $\left\{x: a_{j}<r(x)<b\right\}$. These approximate solutions have been studied extensively by FRID \& SHELUKHIN [2], who derive a number of a priori bounds, including bounds for the mass, energy, and entropy,

$$
\int \rho^{j} d x, \int \rho^{j} E^{j} d x, \int \rho^{j} S\left(\rho^{j}, \mathbf{U}^{j}, \theta^{j}\right) d x \leqq C,
$$

where $C$ is independent of $a_{j}$, as well as a number of pointwise and higher order regularity bounds which do depend on $a_{j}$. Among the latter are pointwise bounds $C_{a_{j}}^{-1} \leqq \rho^{j} \leqq C_{a_{j}}$, established in a rather clever and elegant version of an argument given earlier by KAZHIKHOV \& SHELUKHIN [5]. In the present work we modify this argument in a nontrivial way to establish pointwise bounds for $\rho^{j}$, independent of $a_{j}$, but only away from the origin of Lagrangean space. Specifically, we define the particle position $r_{h}^{j}(t)$ by

$$
h=\int_{a_{j}}^{r_{h}^{j}(t)} \rho^{j}(r, t) r^{m} d r
$$


for given $h \geqq 0$, and we show that, for $h>0$ and $T>0$, there is a constant $C=C(h, T)$ such that

$$
C(h, T)^{-1} \leqq \rho^{j}(x, t) \leqq C(h, T)
$$

for $t \leqq T$ and $r(x) \geqq r_{h}^{j}(t)$. These pointwise bounds then serve as the starting point for the derivation of higher-order regularity estimates for $\mathbf{U}^{j}$ and $\theta^{j}$ away from $t=0$. The bounding constants in these estimates are independent of $a_{j}$, as in (20), but the estimates hold in regions $\left\{x: r(x) \geqq r_{h}^{j}(t)\right\}$ which do depend on $a_{j}$. On the other hand, $\frac{\partial r_{h}^{j}}{\partial t}=u^{j}\left(r_{h}^{j}, t\right)$, so that uniform estimates for $\mathbf{U}^{j}$ imply the convergence as $a_{j} \rightarrow 0$ (ignoring subsequences) of $r_{h}^{j}(\cdot)$ to Hölder-continuous curves $r_{h}(\cdot)$, for $h>0$. Since $r_{h}(t)$ is increasing in $h$, the limit

$$
\underline{r}(t)=\lim _{h \rightarrow 0} r_{h}(t)
$$

exists, and we can then define the "fluid region" $\mathcal{F}$ as in (a) of Theorem 1.1. As asserted in the theorem, $\underline{r}$ is semicontinuous, so that $\mathcal{F} \cap\{t>0\} \cap\{r<b\}$ is an open set. The sequences $\left\{\mathbf{U}^{j}\right\}$ and $\left\{\theta^{j}\right\}$ are then uniformly Hölder continuous on compact subsets of $\mathcal{F} \cap\{t>0\}$, and so have subsequences converging strongly, locally in $\mathcal{F} \cap\{t>0\}$. The pointwise bounds (20) yield weak limits $\rho^{j}(\cdot, t) \rightarrow \rho(\cdot, t)$ on $\{x: \underline{r}(t)+\eta \leqq r(x) \leqq b\}$ for $\eta>0$, and it can be shown as well that $\rho^{j}(\cdot, t) \rightarrow 0$ in $L^{1}(\{x: r(x) \leqq \underline{r}(t)\})$. These limiting arguments, together with the fact that the approximations $\left(\rho^{j}, \mathbf{U}^{j}, \theta^{j}\right)$ are weak solutions, then enable us to conclude that $(\rho, \mathbf{U}, \theta)$ is a weak solution as described in (b)-(f) of Theorem 1.1.

We do not have sufficient information to infer that $\underline{r}(t) \equiv 0$, nor do we know whether solutions exist for which $\underline{r} \not \equiv 0$. The analysis simply shows that $\underline{r}(t)$ may be positive, and that, if it is, a vacuum state of radius $\underline{r}(t)$ centered at the origin or axis of symmetry has emerged. In any case, the total mass is conserved in the spherical case, as is clear from (c) of Theorem 1.1, as is the total mass in any finite cylinder in the cylindrical case. The total momentum is zero, of course, because of the symmetry.

We show in (e) only that the energy equation holds on the support of $\rho$, rather than in the entire space-time domain $\Omega \times(0, \infty)$. This is partly due to the nonstandard characterization of the viscosity terms as distributions in the weak form (17) of the momentum equation, making an interpretation of the $|\nabla \mathbf{U}|^{2}$ mechanical work terms in the energy equation (3) problematic. We may regard the restriction in (e) that the test function be supported in $\mathcal{F}$ as reasonable, since there is no fluid outside $\mathcal{F}$, and the model is not really valid there. On the other hand, this failure to establish the weak form of the energy equation in the entire space-time domain suggests that significant difficulty can be anticipated in extending these results to more general, nonsymmetric flows, in which vacuum states may presumably arise spontaneously at unpredictable points of space-time. Additionally, the failure of the analysis to detect whether or not energy is lost ((f) of Theorem 1.1) calls into question the adequacy of the mass, energy, and entropy bounds (19), which are the only noncontingent a priori bounds known to hold in the multidimensional case 
with energy-class initial data, to yield a satisfactory understanding of the solution operator for these equations.

The theorem of the present paper extends the results of HoFF [3] for the barotropic case, in which $P=P(\rho)$ and the energy equation is omitted, in that more general initial data and completely general velocity directions may occur, and most important, that heat-conduction and temperature-dependent equations of state are allowed. In the simpler barotropic case, the curve $\underline{r}$ is known to be Hölder continuous, and when vacuum states do occur, they occupy open sets in space-time, in which the velocity satisfies a simple homogeneous elliptic equation. As indicated in the discussion above, the situation is considerably more complicated in the nonbarotropic case considered here. Other global existence results for the system (1)-(3) are given in HOFF [4] for two- and three-dimensional flows with nonsymmetric but small initial data, and by Lions (see LIONS [6], FEIREISL [1], and the references in these papers) for the corresponding barotropic system with $P(\rho)=K \rho^{\gamma}$ for certain $\gamma$, and for large data. As far as we are aware, there are no large-data existence results, other than those of the present paper, for the full nonbarotropic system (1)-(3) in a truly multidimensional setting.

The plan of this paper is as follows. First, in Section 2 we derive the pointwise bounds (20) as consequences of the energy and entropy estimates (19) by modifying in a nontrivial way the argument given in FRID \& SHELUKHIN [2]. Then in Section 3 we apply these pointwise bounds to derive a number of higher-order energy estimates for $\mathbf{U}$ and $\theta$ away from $t=0$ and away from the origin of Lagrangean space. These bounds are then applied to establish certain uniform integrability properties of the approximate solutions (it is here that the $L^{q}$ conditions in (12) and (14) play a role). Finally in Section 4 we give the details of the convergence arguments outlined above, showing as well that the limiting functions are indeed weak solutions in the required sense. We note that the final steps of this argument provide a sort of a posteriori validation that the equations (6)-(10) are indeed the correct forms of the general system (1)-(3) in the symmetric cases considered here.

\section{Entropy and pointwise bounds}

In this section we derive a priori pointwise bounds for smooth solutions ( $\rho^{a}, u^{a}$, $\left.v^{a}, w^{a}, \theta^{a}\right)$ of (6)-(10) in the truncated region $0<a<r(x)<b$, together with the additional boundary conditions $u^{a}=\theta_{r}^{a}=0$ at $r=a$ in the spherically symmetric case (for which $v^{a}=w^{a} \equiv 0$ ), and $u^{a}=v^{a}=w_{r}^{a}=\theta_{r}^{a}=0$ at $r=a$ in the cylindrically symmetric case. We assume that the initial data and force are smooth and satisfy the bounds (12)-(14) with constants which are independent of $a$. See the introduction to Section 4 for a brief discussion of the existence of these solutions. As discussed in Section 1, we shall eventually take a sequence of inner radii $a_{j} \rightarrow 0$. Since $a>0$ is fixed for the time being, we suppress the dependence on $j$.

In Lemma 1 below we state the standard energy and entropy estimates for these approximate solutions and in Lemma 2 we derive pointwise bounds for $\rho^{a}$ and $\theta^{a}$. 
Lemma 1. Let $\left(\rho^{a}, u^{a}, v^{a}, w^{a}, \theta^{a}\right)$ be a smooth solution of (6)-(10) defined on $[a, b] \times[0, T]$ with boundary conditions at $r=a$ as described above. Then there are constants $C_{0}$ and $C(T)$ such that

$$
\begin{array}{r}
\int_{a}^{b} \rho^{a}(r, t) r^{m} d r \leqq C_{0}, \\
\int_{a}^{b}\left(\rho^{a} E^{a}\right)(r, t) r^{m} d r \leqq C(T), \\
\int_{a}^{b}\left(\rho^{a} S^{a}\right)(r, t) r^{m} d r+\int_{0}^{t} \int_{a}^{b}\left[\kappa\left(\frac{\theta_{r}^{a 2}}{\theta^{a 2}}\right)+\frac{Q}{\theta^{a}}\right] r^{m} d r d t \leqq C(T)
\end{array}
$$

for all $t \in[0, T]$. Here $E^{a}=\frac{1}{2} \rho\left[u^{a 2}+v^{a 2}+w^{a 2}\right]+\theta^{a}, S$ is the entropy density defined in (15), and $Q$ is as in (11).

Proof. These bounds are standard and follow directly from the equations (6)-(10), the boundary conditions, and the assumptions (12) on the external force.

In order to obtain limiting solutions as $a \rightarrow 0$, we will need further higherorder bounds which are uniform in $a$. These will be obtained away from the origin of Lagrangean space in the following sense. Define curves $r_{h}^{a}(t)$ for $h \geqq 0$ by

$$
h=\int_{a}^{r_{h}^{a}(t)} \rho^{a}(r, t) r^{m} d r
$$

so that, by (6),

$$
\frac{\partial r_{h}^{a}}{\partial t}=u^{a}\left(r_{h}^{a}, t\right)
$$

Thus $r_{h}^{a}(t)$ is the position at time $t$ of a fixed fluid particle. Furthermore, an easy estimate based on Jensen's inequality and the bound in (23) for $\int_{a}^{b} \rho^{a} \Psi\left(1 / \rho^{a}\right) r^{m} d r$ (see (15)) shows that $h \rightarrow 0$ at a uniform rate as $r_{h}^{a}(t) \rightarrow 0$. That is, given $h>0$ there is a positive constant $C=C(h)$ such that

$$
r_{h}^{a}(t) \geqq C(h)^{-1}
$$

independently of $a$ and $T$.

In the following lemma we derive pointwise bounds for $\rho^{a}$ and $\theta^{a}$. The idea of the proof is essentially that of a similar result of FRID \& SHELUKHIN [2], but with a fairly nontrivial modification required to obtain bounds which are independent of $a$.

Lemma 2. Given $h>0$ and $T>0$, there is a constant $C=C(h, T)$, which is independent of $a$, such that, if $r_{h}^{a}(t)$ is as in (24), then

$$
C^{-1} \leqq \rho^{a}(r, t) \leqq C
$$


for $r \in\left[r_{h}^{a}(t), b\right]$ and $t \in[0, T]$, and

$$
\int_{0}^{t}\left\|\theta^{a}(\cdot, \tau)\right\|_{h, \infty} d \tau \leqq C,
$$

where $\|\cdot\|_{h, \infty}$ denotes the sup-norm over $\left[r_{h}^{a}(t), b\right]$.

We first establish the following technical result.

Lemma 3. Fix $h>0$ and $T>0$ and define

$$
\begin{aligned}
F & :=v u_{\xi}^{a}-P^{a}, \\
g(r, t) & :=\int_{r_{h}^{a}(t)}^{r}\left(\frac{\rho^{a}}{y}\left(m u^{a 2}-v^{a 2}\right)-\rho^{a} f_{1}\right)(y, t) d y, \\
\Sigma & :=\rho^{a} u^{a}-F+g, \\
\Phi(r, t) & :=\int_{r_{h}^{a}(0)}^{r} \rho_{0}^{a}(s) u_{0}^{a}(s) d s-\int_{0}^{t} \Sigma(r, \tau) d \tau .
\end{aligned}
$$

Then there exists a constant $C=C(h, T)$, which is independent of $a$, such that

$$
|\Phi(r, t)| \leqq C(h, T) \quad \text { for } r \in\left[r_{h}^{a}(t), b\right], \quad t \in[0, T] .
$$

Proof. We drop the superscript $a$ and observe that

$$
(\rho u)_{t}+\Sigma_{r}=0
$$

and

$$
\Phi_{r}=\rho u, \quad \Phi_{t}=-\Sigma=-\rho u^{2}+F-g .
$$

It follows that

$$
\Phi_{t}+u \Phi_{r}=F-g .
$$

Next let $r(t)$ denote another particle path, defined by

$$
\frac{d r(t)}{d t}=u(r(t), t)
$$

to the right of $r_{h}(t)$. Then

$$
\Phi(r(t), t)=\Phi(r(0), 0)+\int_{0}^{t} F(r(\tau), \tau) d \tau-\int_{0}^{t} g(r(\tau), \tau) d \tau .
$$

The first term on the right is clearly bounded by $C(h, T)$, and for the last term we have

$$
\begin{aligned}
|g(r, \tau)| & =\left|\int_{r_{h}(\tau)}^{r}\left(\frac{\rho}{y}\left(m u^{2}-v^{2}\right)-\rho f_{1}\right)(y, \tau) d y\right| \\
& \leqq C(h, T)\left|\int_{r_{h}(\tau)}^{r} \rho\left(u^{2}+v^{2}+|\mathbf{F}|\right) y^{m} d y\right| \leqq C(h, T)
\end{aligned}
$$


for any $(r, \tau)$ with $r \in\left[r_{h}(\tau), b\right]$, by the energy estimate (22) and the assumption (12) on the force term. The last term in (29) is thus bounded by $C(h, T)$.

The boundedness of the remaining term in (29) requires more work. First observe that we can add an arbitrary function of $t$ to $F$ without changing (28). We consider the function

$$
F(r, t)-\bar{F}(t)
$$

where $\bar{F}(t)$ is the spatial average of $F(\cdot, t)$,

$$
\bar{F}(t):=\frac{n}{b^{n}-a^{n}} \int_{a}^{b} F(r, t) r^{m} d r .
$$

This average is a bounded quantity at each time:

$$
|\bar{F}(t)|=\frac{n}{b^{n}-a^{n}}\left|\int_{a}^{b}\left(\nu u_{\xi}-P\right) r^{m} d r\right|=\frac{n}{b^{n}-a^{n}} \int_{a}^{b} \operatorname{Pr}^{m} d r \leqq C(T)
$$

by the boundary condition for $u$ and the energy estimate (22). Next we estimate the time integral of $F-\bar{F}$ along a particle path $r(t)$ to the right of $r_{h}(t)$ :

$$
\begin{gathered}
\frac{b^{n}-a^{n}}{n} \int_{0}^{t}[F(r(\tau), \tau)-\bar{F}(\tau)] d \tau=\int_{0}^{t} \int_{a}^{b}[F(r(\tau), \tau)-F(r, \tau)] r^{m} d r d \tau \\
=\int_{0}^{t} \int_{a}^{b} r^{m}\left\{\int_{r}^{r(\tau)} F_{y}(y, \tau) d y\right\} d r d \tau \\
=\int_{0}^{t} \int_{a}^{b} r^{m}\left\{\int _ { r } ^ { r ( \tau ) } \left[(\rho u)_{t}+\left(\rho u^{2}\right)_{y}\right.\right. \\
\left.\left.\quad+\frac{m \rho u^{2}}{y}-\frac{\rho v^{2}}{y}-\rho f_{1}\right](y, \tau) d y\right\} d r d \tau \\
=\int_{a}^{b} r^{m} \int_{0}^{t}\left\{\int_{r}^{r(\tau)}(\rho u)_{t} d y+\left[\rho u^{2}\right]_{r}^{r(\tau)}\right\} \\
\left.+\int_{r}^{r(\tau)}\left(\frac{m \rho u^{2}}{y}-\frac{\rho v^{2}}{y}-\rho f_{1}\right)(y, \tau) d y\right\} d \tau d r \\
=\int_{a}^{b} r_{0}^{t}\left\{\frac{d}{d \tau}\left(\int_{r}^{r(\tau)}(\rho u)(y, \tau) d y\right)-\left(\rho u^{2}\right)(r, \tau)\right. \\
\left.+\int_{r}^{r(\tau)}\left(\frac{m \rho u^{2}}{y}-\frac{\rho v^{2}}{y}-\rho f_{1}\right)(y, \tau) d y\right\} d \tau d r .
\end{gathered}
$$

We consider each term in turn. For the first term on the right-hand side of (31) we have

$$
\begin{aligned}
& \left|\int_{a}^{b} r^{m} \int_{0}^{t}\left\{\frac{d}{d \tau} \int_{r}^{r(\tau)}(\rho u)(y, \tau) d y\right\} d \tau d r\right| \\
& \quad \leqq \int_{a}^{b} r^{m}\left|\int_{r}^{r(t)}(\rho u)(y, t) d y\right| d r+\int_{a}^{b} r^{m}\left|\int_{r}^{r(0)}\left(\rho_{0} u_{0}\right)(y) d y\right| d r .
\end{aligned}
$$


The second term here is bounded by $C(h, T)$ by the assumptions (13)-(14) on the initial data. The same bound holds for the first term: for $r<r(t)$ we replace $r^{m}$ by $y^{m}$, while for $r(t)<r$ we use the fact that $y^{-m}<C(h, T)$. This takes care of the first term on the right-hand side of (31), and the energy estimate (22) shows that the second term is bounded by $C(h, T)$ as well. For the third term, on the other hand, the bound (30) does not apply because $r$ now varies over all of $[a, b]$. Instead we split the $y$-integration and interchange the order of $r$ and $y$ integrations to obtain

$$
\begin{aligned}
&\left|\int_{a}^{b} r^{m} \int_{0}^{t} \int_{r}^{r(\tau)} \frac{m\left(\rho u^{2}\right)(y, \tau)}{y} d y d \tau d r\right| \\
& \leqq\left|\int_{0}^{t} \int_{a}^{r(\tau)} \int_{r}^{r(\tau)} m r^{m} \frac{\left(\rho u^{2}\right)(y, \tau)}{y} d y d r d \tau\right| \\
&+\left|\int_{0}^{t} \int_{r(\tau)}^{b} \int_{r(\tau)}^{r} m r^{m} \frac{\left(\rho u^{2}\right)(y, \tau)}{y} d y d r d \tau\right| \\
&= \int_{0}^{t} \int_{a}^{r(\tau)} \int_{a}^{y} m r^{m} \frac{\left(\rho u^{2}\right)(y, \tau)}{y} d r d y d \tau \\
&+\int_{0}^{t} \int_{r(\tau)}^{b} \int_{y}^{b} m r^{m} \frac{\left(\rho u^{2}\right)(y, \tau)}{y} d r d y d \tau \\
& \leqq \frac{m}{n} \int_{0}^{t} \int_{a}^{b}\left(\frac{\left.y^{n}-a^{n}\right)\left(\rho u^{2}\right)(y, \tau) d y d \tau}{y}\right) \\
&+\frac{m}{n} \int_{0}^{t} \int_{r(\tau)}^{b}\left(\frac{b^{n}-y^{n}}{y}\right)\left(\rho u^{2}\right)(y, \tau) d y d \tau \\
& \leqq \frac{m}{n} \int_{0}^{t} \int_{a}^{b}\left(\rho u^{2}\right)(y, \tau) y^{m}+C(h, T) \int_{0}^{t} \int_{r(\tau)}^{b}\left(\rho u^{2}\right)(y, \tau) y^{m} d y d \tau \\
& \leqq C(h, T),
\end{aligned}
$$

where we have used the energy estimate (22) and the fact that $r(t) \geqq r_{h}(t)$. The fourth term on the right-hand side of (31) is treated in the same way, and the last term is bounded by the assumptions (12) on the external force. It follows that

$$
\left|\int_{0}^{t} F(r(\tau), \tau)-\bar{F}(\tau) d \tau\right| \leqq C(h, T) .
$$

Combining this with the fact that $|\bar{F}(\tau)| \leqq C(T)$, we then obtain

$$
\left|\int_{0}^{t} F(r(\tau), \tau) d \tau\right| \leqq C(h, T) .
$$

This shows that the the second term on the right-hand side of (29) is bounded by $C(h, T)$, so that

$$
|\Phi(r, t)| \leqq C(h, T), \quad \text { for } r \geqq r_{h}(t), 0 \leqq t \leqq T .
$$


Proof of Lemma 2. First we establish the upper bound for $\rho$. Defining $L=\log \rho$, we find from (6) that

$$
\dot{L}=-u_{\xi} \leqq-\frac{F}{v}
$$

where $F$ is defined as in the statement of Lemma 3. It follows from (32) that

$$
L(r(t), t) \leqq L(r(0), 0)-\frac{1}{v} \int_{0}^{t} F(r(\tau), \tau) d \tau \leqq C(h, T),
$$

where again $r(t)$ is a particle path to the right of $r_{h}(t)$, and $r_{h}(t)$ is as in (25). This proves that $\rho(r, t)$ is bounded above for $r \geqq r_{h}(t)$.

Next we prove the bound (27) for $\theta$ and the lower bound for $\rho$. Define $H(\Phi)=$ $\exp \left(v^{-1} \Phi\right)$, so that

$$
D_{t}[\rho H(\Phi)]=\left(-u_{\xi}+v^{-1}(F-g)\right)[\rho H(\Phi)]=-v^{-1}(P+g)[\rho H(\Phi)] .
$$

Note that, since $\Phi$ is bounded below, $H(\Phi)>0$ is bounded away from zero, and we can differentiate its reciprocal,

$$
D_{t}\left[\frac{1}{\rho H(\Phi)}\right]=\frac{v^{-1}(P+g)}{\rho H(\Phi)} .
$$

We substitute $P=K \rho \theta$ and apply (30) and the fact that $H(\Phi)^{-1} \leqq C(h, T)$ to obtain

$$
D_{t}\left[\frac{1}{\rho H(\Phi)}\right] \leqq C(h, T)\left(\|\theta(\cdot, t)\|_{h, \infty}+\frac{1}{\rho H(\Phi)}\right) .
$$

Integrating, we get

$$
\left.\frac{1}{\rho H(\Phi)}\right|_{(r, t)} \leqq C(h, T)[1+I(t)]
$$

where

$$
I(t):=\int_{0}^{t}\|\theta(\cdot, \tau)\|_{h, \infty} d \tau .
$$

Since $\Phi$ is bounded above, so is $H(\Phi)$, and we conclude that

$$
\left\|\rho^{-1}(\cdot, t)\right\|_{h, \infty} \leqq C(h, T)[1+I(t)] .
$$

Now, in general, for any two points $x, y$ with $r_{h}(t) \leqq y<x \leqq b$ we have

$$
\begin{aligned}
\theta(x, t)^{1 / 2} \leqq & \theta(y, t)^{1 / 2} \\
& +C(h, T)\left\|\frac{1}{\rho(\cdot, t)}\right\|_{h, \infty}^{1 / 2}\left(\int_{a}^{b} \frac{\theta_{r}^{2}}{\theta^{2}} r^{m} d r\right)^{1 / 2}\left(\int_{a}^{b} \rho \theta r^{m} d r\right)^{1 / 2} .
\end{aligned}
$$


Defining

$$
B(t):=\int_{a}^{b} \frac{\theta_{r}^{2}}{\theta^{2}} r^{m} d r
$$

squaring the above inequality and applying the energy estimate (22), we thus obtain

$$
\theta(x, t) \leqq C(h, T)\left[\theta(y, t)+B(t)\left\|\rho^{-1}(\cdot, t)\right\|_{h, \infty}\right] .
$$

Multiplying through by $y^{m} \rho(y, t)$, integrating with respect to $y$, and using the mass and energy estimates (21) and (22), we get

$$
I^{\prime}(t)=\|\theta(\cdot, t)\|_{h, \infty} \leqq C(h, T)\left[1+B(t)\left\|\rho^{-1}(\cdot, t)\right\|_{h, \infty}\right] .
$$

It then follows from (33) that

$$
I^{\prime}(t) \leqq C(h, T)[1+B(t)(1+I(t))] .
$$

Finally, applying Grönwall's inequality and the fact that

$$
\int_{0}^{t} B(\tau) d \tau \leqq C(T)
$$

which follows from the entropy estimate (23), we conclude that $I(t) \leqq C(h, T)$. This proves (27) and together with (33) implies that $\rho(r, t) \geqq C(\mu, T)^{-1}$ for $r \in\left[r_{h}(t), b\right]$.

\section{Energy estimates and uniform integrability}

Throughout this section $a>0$ will be fixed, and the approximate solutions $\left(\rho^{a}, u^{a}, v^{a}, w^{a}, \theta^{a}\right)$ described at the beginning of Section 2 will be denoted simply by $(\rho, u, v, w, \theta)$.

We shall make repeated use of a cut-off function which is convected with the flow and which vanishes near the origin. To construct such a function we first observe from (32) and (26) that, for $h>0$,

$$
\left|\int_{t_{1}}^{t_{2}} u_{r}\left(r_{h}(t), t\right) d t\right| \leqq C(h, T), \quad 0 \leqq t_{1}, t_{2} \leqq T .
$$

Thus for given $a$ and $h$, we can fix a smooth, increasing function $\phi_{0}(r)$ with $\phi_{0}(r) \equiv$ 0 on $\left[0, r_{h}(0)\right]$ and $\phi_{0}(r) \equiv 1$ on $\left[2 r_{h}(0), b\right]$, and then define $\phi(r, t)$ to be the solution of

$$
\phi_{t}+u \phi_{r}=0
$$

with data $\phi(r, 0)=\phi_{0}(r)$. We choose $\phi_{0}$ so that

$$
\phi_{0}^{\prime}(r) \leqq C(h) \phi_{0}^{(p-1) / p}
$$


for some $p>2$. We shall show that this bound persists for all time. First, for any $\beta \neq 0$ we have

$$
\left(\phi^{\beta} \phi_{r}\right)_{t}+u\left(\phi^{\beta} \phi_{r}\right)_{r}=-\phi^{\beta} \phi_{r} u_{r} .
$$

Integrating along a particle path $r(t)$ to the right of $r_{h}(t)$ (recall the definition (24)) and applying (32), we get

$$
\left|\left(\phi^{\beta} \phi_{r}\right)(r(t), t)\right| \leqq C(h, T)\left|\left(\phi_{0}^{\beta} \phi_{0}^{\prime}\right)(r(0))\right| .
$$

Letting $\beta=(1-p) / p$ and applying (35), we then conclude that

$$
\left|\phi_{r}(r(t), t)\right| \leqq C(h, T) \phi(r(t), t)^{(p-1) / p} .
$$

We shall take $p$ large so that the exponent on the right here is close to one. Notice that we have suppressed here the dependence of $\phi$ on $a$ and $h$.

Next we introduce three higher-order functionals for a given solution: let $\sigma(t)=$ $\min (1, t)$ and define

$$
\begin{aligned}
& \mathcal{A}(T):=\sup _{0 \leqq t \leqq T} \sigma(t) \int_{r_{h}(t)}^{b} \phi(r, t)\left(u_{\xi}^{2}+v_{\xi}^{2}+w_{r}^{2}\right)(r, t) r^{m} d r \\
& +\int_{0}^{T} \int_{r_{h}(t)}^{b} \sigma(t) \phi(r, t)\left[\dot{u}^{2}+\dot{v}^{2}+\dot{w}^{2}\right] r^{m} d r d t, \\
& \mathcal{B}(T):=\sup _{0 \leqq t \leqq T} \sigma(t) \int_{r_{h}(t)}^{b} \phi(r, t) \theta(r, t)^{2} r^{m} d r \\
& +\int_{0}^{T} \int_{r_{h}(t)}^{b} \sigma(t) \phi(r, t) \theta_{r}(r, t)^{2} r^{m} d r d t, \\
& \mathcal{D}(T):=\sup _{0 \leqq t \leqq T} \sigma(t)^{2} \int_{r_{h}(t)}^{b} \phi(r, t)^{2} \theta_{r}(r, t)^{2} r^{m} d r \\
& +\int_{0}^{T} \int_{r_{h}(t)}^{b} \sigma(t)^{2} \phi(r, t)^{2} \dot{\theta}(r, t)^{2} r^{m} d r d t,
\end{aligned}
$$

where we have again suppressed the dependence on $a$ and $h$, and where the dot denotes the convective derivative $\partial_{t}+u \partial_{r}$.

The next lemma is the first step in deriving bounds for $\mathcal{A}, \mathcal{B}$, and $\mathcal{D}$ :

Lemma 4. Let $h>0$ and $T>0$ be given. Then there is a constant $C=C(h, T)$ such that

$$
\int_{0}^{T} \int_{r_{2 h}(t)}^{b}\left(\rho \theta^{2}+u_{\xi}^{2}+v_{\xi}^{2}+w_{r}^{2}\right)(r, t) r^{m} d r d t \leqq C(h, T) .
$$

Proof. For the first integral we have

$$
\int_{0}^{T} \int_{r_{2 h}(t)}^{b} \rho \theta^{2}(r, t) r^{m} d r d t \leqq \int_{0}^{T}\|\theta(t)\|_{\infty, h} \int_{r_{2 h}(t)}^{b} \rho \theta r^{m} d r d t \leqq C(h, T)
$$


by (27) and the energy bound (22). Next, multiplying (7) by $\phi u$ and integrating, we obtain

$$
\begin{aligned}
& \left.\int_{r_{h}(t)}^{b} \frac{1}{2} \rho \phi u^{2} r^{m} d r\right|_{0} ^{t}+v \int_{0}^{t} \int_{r_{h}(t)}^{b} \phi u_{\xi}^{2} r^{m} d r d t \\
& =\int_{0}^{t} \int_{r_{h}(t)}^{b}\left[\frac{\phi \rho u v^{2}}{r}-\phi u P_{r}+\rho \phi u f_{1}-v \phi_{r} u u \xi\right] r^{m} d r d t .
\end{aligned}
$$

Taking $p>2$ in (36), we can bound the pressure term as follows:

$$
\begin{aligned}
- & \int_{0}^{t} \int_{r_{h}(t)}^{b} \phi u P_{r} r^{m} d r d t=K \int_{0}^{t} \int_{r_{h}(t)}^{b} \rho \theta\left(\phi_{r} u+\phi u_{\xi}\right) r^{m} d r d t \\
\leqq & C(h, T) \int_{0}^{t} \int_{r_{h}(t)}^{b}\left(\phi^{1 / 2} \rho u \theta+\rho \phi \theta\left|u_{\xi}\right|\right) r^{m} d r d t \\
\leqq & C(h, T)\left[\int_{0}^{T} \int_{r_{h}(t)}^{b}\left(\rho u^{2}+\rho \theta^{2}\right)(r, t) r^{m} d r d t\right. \\
& \left.+\frac{\delta}{2} \int_{0}^{t} \int_{r_{h}(t)}^{b} \phi u_{\xi}^{2} r^{m} d r d t+\frac{1}{2 \delta} \int_{0}^{T} \int_{r_{h}(t)}^{b} \rho \theta^{2}(r, t) r^{m} d r d t\right] \\
\leqq & C(h, T, \delta)+C(h, T) \delta \int_{0}^{t} \int_{r_{h}(t)}^{b} \phi u_{\xi}^{2} r^{m} d r d t,
\end{aligned}
$$

where we have applied (26) and (40). The last term on the right-hand side of (41) is treated in a similar way, and the force term is easily seen to be bounded by $C(h, T)$. Applying the same analysis to $v$ and $w$, summing the results, and choosing $\delta$ small, we obtain

$\int_{r_{h}(t)}^{b} \frac{1}{2} \rho \phi\left(u^{2}+v^{2}+w^{2}\right) r^{m} d r+\int_{0}^{t} \int_{r_{h}(t)}^{b} \phi\left(u_{\xi}^{2}+v_{\xi}^{2}+w_{r}^{2}\right) r^{m} d r d t \leqq C(h, T)$, which proves the result, since $\phi \equiv 1$ on $\left[r_{2 h}(t), b\right]$.

\subsection{Bound for $\mathcal{A}$}

In this and the following two subsections, $h>0$ and $T>0$ will be fixed, and unless otherwise stated, all integrations in $r$ will be over $\left[r_{h}(t), b\right]$ and will be with respect to $d r$, and all integrations in $t$ will be over $[0, T]$.

To derive a bound for $\mathcal{A}$ we recall (7):

$$
\rho \dot{u}-\frac{\rho v^{2}}{r}+P_{r}=v u_{\xi r}+\rho f_{1} .
$$

Multiplying by $\sigma \phi \dot{u} r^{m}$ and integrating, where again $\sigma(t)=\min (1, t)$, we obtain

$$
\begin{gathered}
\iint\left[\sigma \phi\left(\rho \dot{u}^{2}-\frac{\rho \dot{u} v^{2}}{r}\right)+\sigma \phi \dot{u} P_{r}\right] r^{m} \\
=\iint\left[\nu \sigma \phi u_{\xi r} \dot{u} r^{m}+\sigma \phi \rho f_{1} \dot{u} r^{m}\right] .
\end{gathered}
$$


312

DAVID HoFF \& HELGE KRISTIAN JENSSEN

Integrating by parts in the first term on the right, we get

$$
\begin{aligned}
\iint \sigma \phi u_{\xi r} \dot{u} r^{m}= & -\iint \sigma u_{\xi}\left(\phi \dot{u} r^{m}\right)_{r} \\
= & -\iint \sigma u_{\xi}\left(\phi_{r} \dot{u}+\phi(\dot{u})_{r}+\frac{m \phi \dot{u}}{r}\right) r^{m} \\
= & -\iint \sigma \phi u_{\xi}(\dot{u})_{\xi} r^{m}+O_{h}\left(\iint \sigma \phi^{(p-1) / p}|\dot{u}|\left|u_{\xi}\right| r^{m}\right) \\
= & -\iint \sigma \phi\left[\left(\frac{1}{2} u_{\xi}^{2}\right)_{t}+u_{\xi}\left(u u_{r}\right)_{\xi}\right] r^{m} \\
& +O_{h}\left(\iint \sigma \phi^{(p-1) / p}|\dot{u}|\left|u_{\xi}\right| r^{m}\right),
\end{aligned}
$$

where $O_{h}(I)$ denotes a term which is bounded by $C(h, T)|I|$. The second summand in the first integral in (43) requires some work. We have

$$
\begin{aligned}
\iint \sigma \phi u_{\xi}\left(u u_{r}\right)_{\xi} r^{m} \\
=\iint \sigma \phi u u_{\xi} u_{r r} r^{m}+\iint \sigma \phi u_{r} u_{\xi}^{2} r^{m} \\
=\iint \sigma \phi\left(u\left(\frac{1}{2} u_{r}^{2}\right)_{r}+\frac{m u^{2} u_{r r}}{r}\right) r^{m}+\iint \sigma \phi u_{r}\left(u_{r}+\frac{m u}{r}\right)^{2} r^{m} \\
=-\iint \sigma\left[\frac{1}{2} u_{r}^{2}\left(\phi u r^{m}\right)_{r}+m\left(\phi u^{2} r^{m-1}\right)_{r} u_{r}\right] \\
+O_{h}\left(\iint \sigma \phi\left(\left|u_{r}\right|^{3}+|u|^{3}\right) r^{m}\right) \\
=-\iint \sigma \phi_{r}\left(\frac{1}{2} u u_{r}^{2} r^{m}+m u^{2} r^{m-1} u_{r}\right) \\
+O_{h}\left(\iint \sigma \phi\left(\left|u_{r}\right|^{3}+|u|^{3}\right) r^{m}\right) \\
=O_{h}\left(\iint \sigma \phi^{(p-1) / p}\left(|u|^{2}\left|u_{r}\right|+|u|\left|u_{r}\right|^{2}\right) r^{m}\right) \\
+O_{h}\left(\iint \sigma \phi\left(\left|u_{r}\right|^{3}+|u|^{3}\right) r^{m}\right) .
\end{aligned}
$$

We thus obtain, from (43),

$$
\begin{aligned}
v \int & \int \sigma \phi u_{\xi r} \dot{u} r^{m} \\
= & -v \iint \sigma \phi\left(\frac{1}{2} u_{\xi}^{2}\right) r_{t} r^{m}+O_{h}\left(\iint \sigma \phi\left(\left|u_{r}\right|^{3}+|u|^{3}\right) r^{m}\right) \\
& +O_{h}\left(\iint \sigma \phi^{(p-1) / p}\left(|u|^{2}\left|u_{r}\right|+|u|\left|u_{r}\right|^{2}+|\dot{u}|\left|u_{\xi}\right|\right) r^{m}\right) .
\end{aligned}
$$


Next consider the first term on the right-hand side of (44). Integrating first with respect to $t$ and applying the equation satisfied by $\phi$, we obtain

$$
\begin{aligned}
& -v \iint \sigma \phi\left(\frac{1}{2} u_{\xi}^{2}\right)_{t} r^{m} \\
& =-\left.\frac{1}{2} v \sigma(T) \int \phi u_{\xi}^{2} r^{m}\right|_{t=T}+\frac{1}{2} v \iint\left(\sigma_{t} \phi-\sigma u \phi_{r}\right) u_{\xi}^{2} r^{m} \\
& =-\left.\frac{1}{2} \nu \sigma(T) \int \phi u_{\xi}^{2} r^{m}\right|_{t=T}+O_{h}\left(1+\iint \sigma \phi^{(p-1) / p}|u||u \xi|^{2} r^{m}\right),
\end{aligned}
$$

where we have used Lemma 4 to bound the term containing $\sigma_{t}$. For the first term on the right-hand side of (42) we thus have

$$
\begin{aligned}
v & \iint \sigma \phi u_{\xi r} \dot{u} r^{m} \\
= & -\left.\frac{1}{2} \nu \sigma(T) \int \phi u_{\xi}^{2} r^{m}\right|_{t=T}+O_{h}\left(1+\iint \sigma \phi\left(\left|u_{r}\right|^{3}+|u|^{3}\right) r^{m}\right. \\
& \left.+\iint \sigma \phi^{(p-1) / p}\left(|u|^{2}\left|u_{\xi}\right|+|u|\left|u_{\xi}\right|^{2}+|u|^{3}+|\dot{u}|\left|u_{\xi}\right|\right) r^{m}\right)
\end{aligned}
$$

since $u_{r}=u_{\xi}+(m u) / r$.

Next we estimate the $P_{r}$ term on the left-hand side of (42):

$$
\begin{aligned}
\int & \int \sigma \phi \dot{u} P_{r} r^{m} \\
= & -\iint \sigma\left(\phi(\dot{u})_{r}+\phi \frac{m \dot{u}}{r}+\phi_{r} \dot{u}\right) P^{m} \\
\leqq & -\iint \sigma \phi\left(u_{r t}+\left(u u_{r}\right)_{r}\right) r^{m}+O_{h}\left(\iint \sigma\left(\phi_{r}|\dot{u} P|+\phi|\dot{u} P|\right) r^{m}\right) \\
= & -\left.\int \sigma \phi u_{r} P r^{m}\right|_{t=T}+\iint(\sigma \phi P)_{t} u_{r} r^{m}+\iint \sigma u u_{r}\left(\phi P r^{m}\right)_{r} \\
& +O_{h}\left(\iint \sigma \phi^{(p-1) / p}|\dot{u}| P^{m}\right) .
\end{aligned}
$$

The second and third terms on the right-hand side of (46) are bounded as follows:

$$
\begin{aligned}
\int & \int(\sigma \phi P)_{t} u_{r} r^{m}+\iint \sigma u u_{r}\left(\phi P r^{m}\right)_{r} \\
= & \iint \sigma_{t} \phi P u_{r} r^{m}+\sigma\left(\phi_{t}+u \phi_{r}\right) u_{r} P r^{m} \\
& +m \iint \sigma \phi u u_{r} P r^{m-1}+\iint \sigma \phi\left(P_{t}+u P_{r}\right) u_{r} r^{m} \\
= & \iint \sigma_{t} \phi P u_{r} r^{m}+m \iint \sigma \phi u u_{r} P r^{m-1} \\
& -K \iint \sigma \phi u_{r} \rho u_{\xi} \theta r^{m}+K \iint \sigma \phi u_{r} \rho \dot{\theta} r^{m} \\
= & O_{h}\left(1+\iint \sigma \phi\left(\theta\left(u^{2}+u_{\xi}^{2}\right)+\left|u_{r} \| \dot{\theta}\right|\right) r^{m}\right),
\end{aligned}
$$


where we have used the equation for $\phi$, Lemma 4, and the pointwise upper bound on the density. Substituting back into (46), we thus obtain

$$
\begin{aligned}
\iint \sigma \phi \dot{u} P_{r} r^{m}= & -\left.\int \sigma \phi u_{r} P r^{m}\right|_{t=T} \\
& +O_{h}\left(1+\iint \sigma \phi\left(\theta\left(u^{2}+u_{r}^{2}\right)+\left|u_{r}\right||\dot{\theta}|\right) r^{m}\right) \\
& +O_{h}\left(\iint \sigma \phi^{(p-1) / p}|\dot{u}| \theta r^{m}\right) .
\end{aligned}
$$

Substituting (45) and (47) into (42) we obtain finally

$$
\begin{aligned}
\iint & \sigma \phi\left(\rho \dot{u}^{2}-\frac{\rho \dot{u} v^{2}}{r}\right) r^{m}+\left.\frac{1}{2} v \sigma(T) \int \phi u_{\xi}^{2} r^{m}\right|_{t=T} \\
= & \left.\sigma(T) \int \phi P u_{r} r^{m}\right|_{t=T}+\iint \sigma \phi \rho f_{1} \dot{u} r^{m} \\
& +O_{h}\left(1+\iint \sigma \phi^{(p-1) / p}\left(|u|^{2} u_{\xi}+|u|\left|u_{\xi}\right|^{2}+|u|^{3}+|\dot{u}|\left|u_{\xi}\right|+|\dot{u}| \theta\right) r^{m}\right. \\
& \left.+\iint \sigma \phi\left(\theta\left(u^{2}+u_{\xi}^{2}\right)+\left|u_{r}\right||\dot{\theta}|+\left|u_{\xi}\right|^{3}+|u|^{3}\right) r^{m}\right) .
\end{aligned}
$$

Treating the variables $v$ and $w$ in a similar way and applying the pointwise bounds (26) for the density, we then obtain

$$
\begin{aligned}
& \iint \sigma \phi \rho|\dot{U}|^{2} r^{m}+\left.\frac{1}{2} \sigma(T) \int \phi|\nabla U|^{2} r^{m}\right|_{t=T} \\
& \leqq C(h, T)\left\{1+\iint \sigma \phi \rho\left|u v \dot{v}-v^{2} \dot{u}\right| r^{m}+\left.\sigma(T) \int \phi P\left|u_{r}\right| r^{m}\right|_{t=T}\right. \\
&+\iint \sigma \phi\left(\theta\left(u^{2}+u_{\xi}^{2}\right)+\left|u_{r}\right||\dot{\theta}|+|U|^{3}+|\nabla U|^{3}\right) r^{m} \\
&\left.+\iint \sigma \phi^{(p-1) / p}\left(|\dot{u}| \theta+|U|^{3}+|U||\nabla U|^{2}+\dot{U} \cdot \nabla U\right) r^{m}\right\}
\end{aligned}
$$

where

$$
\begin{aligned}
& U=(u, v, w), \quad|\dot{U}| \equiv|\dot{u}|+|\dot{v}|+|\dot{w}|, \\
& |\nabla U|^{2} \equiv\left|u_{\xi}\right|^{2}+\left|v_{\xi}\right|^{2}+\left|w_{r}\right|^{2}, \\
& F=\left(f_{1}, f_{2}, f_{3}\right), \quad|F|=\left|f_{1}\right|+\left|f_{2}\right|+\left|f_{3}\right| .
\end{aligned}
$$

A fairly lengthy analysis is required to bound each of the terms on the right-hand side here. For example the $u v \dot{v}$ term is

$$
\iint \sigma \phi \rho|u v \dot{v}| r^{m} \leqq \frac{1}{2} \delta \iint \sigma \phi \rho|\dot{v}|^{2} r^{m}+C(\delta) \iint \sigma \phi \rho\left(|u|^{4}+|v|^{4}\right) r^{m}
$$


Choosing $\delta$ small, we absorb the first term here into the left-hand side of (48). Also,

$$
\begin{aligned}
\iint \sigma \phi|u|^{4} r^{m} & \leqq \int\left(\|u(\cdot, t)\|_{\infty, h}^{2} \int \rho|u|^{2} r^{m}\right) \\
& \leqq C(h, T) \iint\left(|u \xi|^{2}+\rho|u|^{2}\right) r^{m} \leqq C(h, T) .
\end{aligned}
$$

We have used here the pointwise bound (26) for $\rho$, the energy estimate (22), and Lemma 4. Applying similar estimates to the other terms in (48), we finally obtain

$$
\begin{aligned}
& \iint \sigma \phi|\dot{U}|^{2} r^{m}+\left.\sigma(T) \int \phi|\nabla U|^{2} r^{m}\right|_{t=T} \\
& \leqq C(h, T)\left\{1+\mathcal{D}(T)^{1 / 2}+\mathcal{A}(T)^{1 / 2}\right. \\
&\left.\quad+\int\|\theta(\cdot, t)\|_{\infty, h} \mathcal{A}(t) d t+\iint \sigma \phi|\nabla U|^{3} r^{m}\right\} .
\end{aligned}
$$

For the $|\nabla U|^{3}$ term on the right here we apply the following Sobolev estimate, which is standard, and whose proof we omit:

Lemma 5. Let $J(r, t)$ be a smooth function defined on $\left\{(r, t) \mid r_{h}(t) \leqq r \leqq b, t \in\right.$ $[0, T]\}$. Then

$$
\begin{aligned}
& \iint \sigma \phi|J|^{3} r^{m} d r d t \\
& \leqq C(h, T) \sup _{0 \leqq t \leqq T}\left(\sigma(t) \int \phi J^{2} r^{m} d r\right)^{1 / 2} \\
& \quad \times\left[\iint J^{2} r^{m} d r d t+\left(\iint \sigma \phi J_{r}^{2} r^{m} d r d t\right)^{1 / 4} \iint\left(J^{2} r^{m} d r d t\right)^{3 / 4}\right] .
\end{aligned}
$$

Taking $J=v_{\xi}$, for example, we have

$$
\iint \sigma \phi\left|v_{\xi}\right|^{3} r^{m} \leqq C(h, T) \mathcal{A}(T)^{1 / 2}\left[1+\left(\iint \sigma \phi v_{\xi r}^{2} r^{m}\right)^{1 / 4}\right],
$$

by the definition (37) of $\mathcal{A}$ and Lemma 4 . To bound the last integral here we square (8), multiply by $\sigma \phi r^{m}$, and perform routine estimates. The result is that

$$
\begin{aligned}
\iint \sigma \phi v_{\xi r}^{2} r^{m} & \leqq C(h, T) \iint \sigma \phi\left(\dot{v}^{2}+|U|^{4}+|F|^{2}\right) r^{m} \\
& \leqq C(h, T)\left(1+\mathcal{A}(T)+\iint \sigma \phi|U|^{4} r^{m}\right) \\
& \leqq C(h, T)(1+\mathcal{A}(T)),
\end{aligned}
$$

where we have used the hypothesis (12) on the external force and the bound in (49). We thus have

$$
\iint \sigma \phi\left|v_{\xi}\right|^{3} r^{m} \leqq C(h, T) \mathcal{A}(T)^{1 / 2}\left(1+\mathcal{A}(T)^{1 / 4}\right) \leqq C(h, T)\left(1+\mathcal{A}(T)^{3 / 4}\right) .
$$


Similar estimates apply to $u_{\xi}$ and $w_{r}$, so that $\mathcal{A}(T) \leqq C(h, T)\left(1+\mathcal{A}(T)^{3 / 4}+\mathcal{B}(T)+\mathcal{D}(T)^{1 / 2}+\int_{0}^{T}\|\theta(\cdot, t)\|_{\infty, h} \mathcal{A}(t) d t\right)$

Applying Grönwall's inequality we finally obtain

$$
\mathcal{A}(T) \leqq C(h, T)\left(1+\mathcal{B}(T)+\mathcal{D}(T)^{1 / 2}\right) .
$$

\subsection{Bound for $\mathcal{B}$}

From (10), (6), and (34) we get

$$
\frac{1}{2} \sigma\left[\left(\phi \rho \theta^{2}\right)_{t}+\left(\phi \rho u \theta^{2}\right)_{\xi}\right]=\sigma \phi \theta\left(\kappa \theta_{r \xi}-P u_{\xi}+Q\right) .
$$

Integrating and using Lemma 4 we obtain

$$
\left.\frac{1}{2} \sigma(T) \int \phi \rho \theta^{2} r^{m}\right|_{t=T} \leqq C(h, T)+\iint \sigma \phi \theta\left(\kappa \theta_{r \xi}-P u_{\xi}+Q\right) r^{m} .
$$

The terms on the right are treated as above in the estimates leading to (50). The result is that

$$
\mathcal{B}(T) \leqq C(h, T)\left(1+\mathcal{D}(T)^{1 / 2}\right)
$$

so that, from (50),

$$
\mathcal{A}(T)+\mathcal{B}(T) \leqq C(h, T)\left(1+\mathcal{D}(T)^{1 / 2}\right) .
$$

\subsection{Bound for $\mathcal{D}$}

Recall the temperature equation (10):

$$
\rho \dot{\theta}=\kappa \theta_{r \xi}-P u_{\xi}+Q .
$$

Multiplying by $\sigma^{2} \phi^{2} \dot{\theta} r^{m}$ and integrating, we obtain

$$
\iint \sigma^{2} \phi^{2} \rho \dot{\theta}^{2} r^{m}=\kappa \iint \sigma^{2} \phi^{2} \theta_{r \xi} \dot{\theta} r^{m}+\iint \sigma^{2} \phi^{2} \dot{\theta}\left(-P u_{\xi}+Q\right) r^{m},
$$

so that

$$
\begin{aligned}
& \iint \sigma^{2} \phi^{2} \dot{\theta}^{2} r^{m}+\left.\frac{1}{2} \sigma(T)^{2} \int \phi^{2} \theta_{r}^{2} r^{m}\right|_{t=T} \\
& \leqq \\
& \leq(h, T, \delta) \iint\left[\sigma \phi^{2} \theta_{r}^{2}+\sigma^{2} \phi^{2}\left(|u| \theta_{r}^{2}+\left|u_{\xi}\right| \theta_{r}^{2}+u_{\xi}^{2} \theta^{2}+Q^{2}\right)\right. \\
& \left.\quad+\sigma^{2} \phi \phi_{r}\left|\dot{\theta} \theta_{r}\right|\right] r^{m},
\end{aligned}
$$


for suitably small $\delta$. We shall estimate the $Q^{2}$ term in some detail. Recalling the definition $F=v u_{\xi}-P$, we get

$$
\begin{aligned}
\iint \sigma^{2} \phi^{2} Q^{2} r^{m} & \leqq C(h, T) \iint \sigma^{2} \phi^{2}\left(u_{\xi}^{4}+v_{\xi}^{4}+w_{r}^{4}+|U|^{4}\right) r^{m} \\
& \leqq C(h, T) \iint \sigma^{2} \phi^{2}\left(F^{4}+\theta^{4}+v_{\xi}^{4}+w_{r}^{4}+|U|^{4}\right) r^{m}
\end{aligned}
$$

For the first term on the right-hand side we note that

$$
F_{r}=\rho \dot{u}-\frac{\rho v^{2}}{r}-\rho f_{1}
$$

by (7), so that, for $p \geqq 2$,

$$
\begin{aligned}
\left\|\phi^{3 / 4} F(\cdot, t)\right\|_{\infty, h}^{2} \\
\quad \leqq \int\left|\frac{3}{2} \phi^{1 / 2} \phi_{r} F^{2}+2 \phi^{3 / 2} F\left(\rho \dot{u}-\frac{\rho v^{2}}{r}-\rho f_{1}\right)\right| \\
\quad \leqq C(h, T) \int\left[\phi^{3 / 2-1 / p}\left(u_{\xi}^{2}+\theta^{2}\right)+\phi^{3 / 2}\left(\left|u_{\xi}\right|+\theta\right)\left(|\dot{u}|+v^{2}+|F|\right)\right] r^{m} \\
\quad \leqq C(h, T) \int\left[\phi\left(u_{\xi}^{2}+\theta^{2}+v^{4}+|F|^{2}\right)+\phi^{3 / 2}\left(\left|u_{\xi}\right|+\theta\right)|\dot{u}|\right] r^{m} .
\end{aligned}
$$

Thus

$$
\begin{aligned}
\int & \int \sigma^{2} \phi^{2} F^{4} r^{m} \\
\leqq & \int \sigma^{2}\left\|\phi^{3 / 4} F(\cdot, t)\right\|_{\infty, h}^{2}\left(\int \phi^{1 / 2} F^{2} r^{m}\right) \\
\leqq & C(h, T) \int \sigma^{2}\left(\int\left[\phi\left(u_{\xi}^{2}+\theta^{2}+v^{4}+|F|^{2}\right)+\phi^{3 / 2}\left(\left|u_{\xi}\right|+\theta\right)|\dot{u}|\right] r^{m}\right) \\
& \times\left(\int \phi^{1 / 2}\left(u_{\xi}^{2}+\theta^{2}\right) r^{m}\right) d t \\
\leqq & C(h, T)\left\{\left[1+\sup _{0 \leqq t \leqq T} \int \sigma \phi\left(u_{\xi}^{2}+\theta^{2}+v^{4}\right) r^{m}\right]\left(\iint \phi^{1 / 2}\left(u_{\xi}^{2}+\theta^{2}\right) r^{m}\right)\right. \\
& +\int \sigma^{2}\left(\int \phi^{2}\left(u_{\xi}^{2}+\theta^{2}\right) r^{m}\right)^{1 / 2}\left(\int \phi \dot{u}^{2} r^{m}\right)^{1 / 2} \\
& \left.\times\left(\int \phi\left(u_{\xi}^{2}+\theta^{2}\right) r^{m}\right)^{1 / 2}\left(\int\left(u_{\xi}^{2}+\theta^{2}\right) r^{m}\right)^{1 / 2} d t\right\} \\
\leqq & C(h, T)\left\{1+\left(\sup _{0 \leqq t \leqq T} \sigma \phi\left(u_{\xi}^{2}+\theta^{2}+v^{4}\right) r^{m}\right)\right. \\
& \left.+\int\left(\sigma \int \phi\left(u_{\xi}^{2}+\theta^{2}\right) r^{m}\right)\left(\int \sigma \phi \dot{u}^{2} r^{m}\right)^{1 / 2}\left(\int\left(u_{\xi}^{2}+\theta^{2}\right) r^{m}\right)^{1 / 2} d t\right\}
\end{aligned}
$$




$$
\begin{aligned}
\leqq & C(h, T)\left\{1+\left(\sup _{0 \leqq t \leqq T} \sigma \int \phi\left(u_{\xi}^{2}+\theta^{2}+v^{4}\right) r^{m}\right)\right. \\
& \left.\times\left(1+\left(\iint \sigma \phi \dot{u}^{2} r^{m}\right)^{1 / 2}\right)\right\} \\
\leqq & C(h, T)\left\{1+\left(1+\mathcal{A}(T)^{1 / 2}\right)\left(\sup _{0 \leqq t \leqq T} \sigma \int \phi\left(u_{\xi}^{2}+\theta^{2}+v^{4}\right) r^{m}\right)\right\} .
\end{aligned}
$$

We have used here the assumption (12) on the force term and the result of Lemma 4. To bound the $v^{4}$ term we argue as follows: from the energy estimate (22) we have

$$
\begin{aligned}
\sigma \int \phi v^{4} r^{m} & \leqq \sigma\left\|\phi v(\cdot, t)^{2}\right\|_{\infty, h}\left(\int v^{2} r^{m}\right) \\
& \leqq C(h, T) \sigma \int\left(\phi_{r} v^{2}+\phi v v_{r}\right) \leqq C(h, T)\left(1+\sigma \int \phi v_{\xi}^{2} r^{m}\right) \\
& \leqq C(h, T) \mathcal{A}(T) .
\end{aligned}
$$

It then follows from the definitions of $\mathcal{A}$ and $\mathcal{B}$ that

$$
\iint \sigma^{2} \phi^{2} F^{4} r^{m} \leqq C(h, T)\left(1+\mathcal{A}(T)^{1 / 2}\right)(1+\mathcal{A}(T)+\mathcal{B}(T)) .
$$

Applying similar arguments to the other terms in (52) and recalling (51), we conclude that

$$
\mathcal{D}(T) \leqq C(T, h)\left(1+\mathcal{D}(T)^{3 / 4}+\int \mathcal{D}(t)\|\theta(\cdot, t)\|_{\infty, h}\right) .
$$

Applying Grönwall's inequality together with (27) we get

$$
\mathcal{D}(T) \leqq C(h, T),
$$

and hence, by (51),

$$
\mathcal{A}(T), \mathcal{B}(T) \leqq C(h, T)
$$

We have proved:

Lemma 6. Let $h>0$ and $T>0$ be given. Then there is a constant $C=C(h, T)$ such that, if $\mathcal{A}, \mathcal{B}$, and $\mathcal{D}$ are as defined above in (37)-(39), then

$$
\mathcal{A}(T), \mathcal{B}(T), \mathcal{D}(T) \leqq C(h, T) .
$$

Next we derive two auxiliary bounds: in Lemma 7 below we prove an $L^{q}$ bound for the approximate velocities, and in Lemma 8 we prove a bound for the time integral of their sup-norms. These auxiliary results will then be applied in Lemma 9 to prove the uniform integrability of the approximate densities and energies. This uniform integrability will be crucial later in Section 4 in showing that limits of these approximate solutions are indeed weak solutions of the original system. 
Lemma 7. There is a constant $C=C(T)$, independent of a, such that, for the cylindrically symmetric case,

$$
\int_{a}^{b} \rho(r, t)\left(|r v(r, t)|^{q}+|w(r, t)|^{q}\right) r d r \leqq C(T) C_{0} \quad \text { for } \quad t \leqq T
$$

where $q>2$ and $C_{0}$ are as in (14).

Proof. Recall that $m=1$ in the cylindrically symmetric case. Denoting $\operatorname{sign}(v)$ by $s$ we have, upon multiplying (8) by $r s$,

$$
\rho\left(|r v|_{t}+u|r v|_{r}\right)=r s\left(\frac{\mu}{r}(r v)_{r}\right)_{r}+s \rho f_{2} r .
$$

Multiplying by $q r|r v|^{q-1}$ and adding $|r v|^{q}\left[(r \rho)_{t}+(r \rho u)_{r}\right]=0$, we then get

$$
\left(r \rho|r v|^{q}\right)_{t}+\left(r \rho u|r v|^{q}\right)_{r}=q r^{2} s|r v|^{q-1}\left(\frac{\mu}{r}(r v)_{r}\right)_{r}+q s \rho f_{2}|r v|^{q-1} r^{2},
$$

so that

$$
\begin{aligned}
& \left.\int \rho|r v|^{q} r d r\right|_{0} ^{t}+\mu q \iint\left[2 s|r v|^{q-1}+(q-1) r s^{2}|r v|^{q-2}(r v)_{r}\right](r v)_{r} d r d t \\
& =q \iint s \rho f_{2}|r v|^{q-1} r^{2} d r d t .
\end{aligned}
$$

Observe that the first summand in the double integral on the left integrates to zero, while the second summand is positive. For the term on the right, we have from (12) that

$$
\begin{aligned}
\iint s \rho f_{2}|r v|^{q-1} r^{2} d r d t & \leqq C \iint\left(\left|f_{2}\right|^{q} \rho r+\rho|r v|^{q} r\right) d r d t \\
& \leqq C\left(1+\iint \rho|r v|^{q} d r d t\right) .
\end{aligned}
$$

The result then follows from Grönwall's inequality and the assumption on $v_{0}$. A similar argument applies to $w$.

The following bounds are consequences of the entropy estimate (23):

Lemma 8. Given $R>0$ and $T>0$, there is a constant $C=C(R, T)$ such that

$$
\int_{t_{1}}^{t_{2}}\left(\left\|\frac{u}{\theta^{1 / 2}}\right\|_{\infty, R}+\|\log (\theta \vee 1)\|_{\infty, R}\right) d t \leqq C(R, T)
$$

for $t_{1}, t_{2} \in[0, T]$. Here $\theta \vee 1=\max (\theta, 1)$. 
Proof. Applying the boundary condition $u(b, t) \equiv 0$, we get, for $r \in[R, b]$,

$$
\begin{aligned}
\left|\frac{u}{\theta^{1 / 2}}(r, t)\right| & \leqq C \int_{r}^{b}\left(\frac{\left|u_{r}\right|}{\theta^{1 / 2}}+\frac{|u|\left|\theta_{r}\right|}{\theta^{3 / 2}}\right) d r \\
& \leqq C(R) \int_{R}^{b}\left(1+\frac{\theta_{r}^{2}}{\theta^{2}}+\frac{u_{r}^{2}+u^{2} / r^{2}}{\theta}\right) r^{m} d r,
\end{aligned}
$$

so that, by (23),

$$
\int_{t_{1}}^{t_{2}}\left\|\frac{u}{\theta^{1 / 2}}\right\|_{\infty, R} d t \leqq C(R) \int_{0}^{T} \int_{R}^{b}\left(1+\frac{\left|\theta_{r}\right|^{2}}{\theta^{2}}+\frac{Q}{\theta}\right) r^{m} d r \leqq C(R, T) .
$$

Next let $\tilde{\theta}=\theta \vee 1$ so that, for $r \in[R, b]$,

$$
\begin{aligned}
\log \tilde{\theta}(r, t) & \leqq \log \tilde{\theta}(b, t)+\int_{r}^{b}\left|\frac{\tilde{\theta}_{r}}{\tilde{\theta}}\right| d r \leqq \log \tilde{\theta}(b, t)+\int_{r}^{b}\left|\frac{\theta_{r}}{\theta}\right| d r \\
& \leqq \log \tilde{\theta}(b, t)+C(R)\left(\int_{R}^{b}\left|\frac{\theta_{r}}{\theta}\right|^{2} r^{m} d r\right)^{1 / 2} .
\end{aligned}
$$

Integrating in time and using the $h$-dependent bound (27) with $h=M_{0} / 2$ for the first term on the right ( $M_{0}$ is the total mass), we conclude from (23) that

$$
\int_{0}^{T}\|\log \tilde{\theta}\|_{\infty, R} d t \leqq C(R, T) .
$$

We now apply the above two lemmas to prove certain uniform integrability estimates. To describe these, we define the strictly increasing, convex function

$$
G:[1, \infty) \rightarrow[0, \infty), \quad G(y):=y \log y .
$$

Then $G^{-1}:[0, \infty) \rightarrow[1, \infty)$, and we can define for $r>0, c>0$ the function

$$
\omega(r ; c):=r+r G^{-1}\left(\frac{c}{r}\right) .
$$

It is easily checked that for each fixed $c$ the function $r \mapsto \omega(r ; c)$ is continuous and increasing on $(0, \infty)$, and that

$$
\lim _{r \downarrow 0} \omega(r ; c)=0 .
$$

Finally, if $E \subset[0, b]$, we define $|E|:=\int_{E} r^{m} d r$.

Lemma 9. Let $\omega$ be as described above.

(a) If $a \geqq 0$ and $\rho:[a, b] \rightarrow \mathbf{R}$ is strictly positive and satisfies

$$
\left|\int_{a}^{b} \rho \log \rho r^{m} d r\right| \leqq C
$$

then for any measurable set $E \subset[a, b]$,

$$
\int_{E} \rho r^{m} d r \leqq \omega(|E| ; C) \text {. }
$$


(b) Let $R>0$ and $T>0$. Then there is a constant $C=C(R, T)$ such that, if $E(t)$ is a measurable subset of $[R, b]$ for each $t \in[0, T]$, and if $(\rho, u, v, w, \theta)=$ $\left(\rho^{a}, u^{a}, v^{a}, w^{a}, \theta^{a}\right)$ is the approximate solution described at the beginning of Section 2, then

$$
\begin{aligned}
\int_{0}^{T} \int_{E(t)} \rho \theta r^{m} d r d t & \leqq \omega\left(\int_{0}^{T} \int_{E(t)} \rho r^{m} d r d t ; C(R, T)\right) \\
\int_{0}^{T} \int_{E(t)} \rho u^{2} r^{m} d r d t & \leqq C(R, T) \omega\left(\int_{0}^{T} \int_{E(t)} \rho r^{m} d r d t ; C(R, T)\right)^{1 / 4} .
\end{aligned}
$$

(c) Let $R, T, E(T), C(R, T)$, and $(\rho, u, v, w, \theta)$ be as in (b) for the cylindrically symmetric case. Then

$$
\int_{0}^{T} E(t) \int \rho(r, t)\left(v^{2}+w^{2}\right) r d r d t \leqq C(R, T)\left(\int_{0}^{T} E(t) \int \rho r d r d t\right)^{(q-2) / q}
$$

where $q>2$ is as in (14).

Proof. To prove (a) we define $E_{1}:=\{r \in E: \rho(r) \leqq 1\}$ and $E_{2}:=\{r \in E$ : $\rho(r)>1\}$. Then

$$
\int_{E_{1}} \rho r^{m} d r \leqq \int_{E_{1}} r^{m} d r \leqq|E|
$$

If $\left|E_{2}\right| \neq 0$, then

$$
\frac{1}{\left|E_{2}\right|} \int_{E_{2}} \rho r^{m} d r \geqq 1
$$

Applying Jensen's inequality to the convex function $G$, we find from (55) that

$$
G\left(\frac{1}{\left|E_{2}\right|} \int_{E_{2}} \rho r^{m} d r\right) \leqq \frac{1}{\left|E_{2}\right|} \int_{E_{2}} G(\rho) r^{m} d r \leqq \frac{C}{\left|E_{2}\right|}
$$

Thus by (54),

$$
\int_{E_{2}} \rho r^{m} d r \leqq\left|E_{2}\right| G^{-1}\left(\frac{C}{\left|E_{2}\right|}\right) \leqq|E| G^{-1}\left(\frac{C}{|E|}\right),
$$

and we conclude that

$$
\int_{E} \rho r^{m} d r=\int_{E_{1}} \rho r^{m} d r+\int_{E_{2}} \rho r^{m} d r \leqq|E|+|E| G^{-1}\left(\frac{C}{|E|}\right)=\omega(|E| ; C) .
$$
Set

To prove (b) we let $\tilde{\theta}=\max \{\theta, 1\}$ and define the mass measure $d m:=\rho r^{m} d r$.

$$
E_{1}(t):=\left\{r \in(E(t): \theta(r, t) \leqq 1\}, \quad E_{2}(t):=\{r \in(E(t): \theta(r, t)>1\} .\right.
$$


Then

$$
\int_{0}^{T} \int_{E_{1}(t)} \theta d m \leqq \int_{0}^{T} \int_{E(t)} d m
$$

Next, if

$$
\int_{0}^{T} \int_{E_{2}(t)} d m>0
$$

then with $G$ as in (53),

$$
\begin{aligned}
G\left(\frac{\int_{0}^{T} \int_{E_{2}(t)} \theta d m d t}{\int_{0}^{T} \int_{E_{2}(t)} d m d t}\right) & \leqq \frac{\int_{0}^{T} \int_{E_{2}(t)} G(\theta) d m d t}{\int_{0}^{T} \int_{E_{2}(t)} d m d t} \\
& =\frac{\int_{0}^{T} \int_{E_{2}(t)} \rho \theta \log \theta r^{m} d r d t}{\int_{0}^{T} \int_{E_{2}(t)} d m d t} \\
& \leqq \frac{\int_{0}^{T}\|\log \tilde{\theta}\|_{\infty, R}\left(\int_{E_{2}(t)} \rho \theta r^{m} d r\right) d t}{\int_{0}^{T} \int_{E_{2}(t)}^{T} d m d t} \\
& \leqq \frac{C(R, T)}{\int_{E_{2}(t)}^{T} d m d t}
\end{aligned}
$$

where we have used the energy estimate (22) and the previous lemma. Thus

$$
\begin{aligned}
\int_{0}^{T} \int_{E_{2}(t)} \rho \theta r^{m} d r d t & \leqq\left(\int_{0}^{T} \int_{E_{2}(t)} d m d t\right) \cdot G^{-1}\left(\frac{C(R, T)}{\int_{0}^{T} \int_{E_{2}(t)} d m d t}\right) \\
& \leqq\left(\int_{0}^{T} \int_{E(t)} d m d t\right) \cdot G^{-1}\left(\frac{C(R, T)}{\int_{0}^{T} \int_{E(t)} d m d t}\right)
\end{aligned}
$$

We have used here the fact that $s \mapsto s G^{-1}(C / s)$ is increasing. Adding this to (56), we obtain the first estimate in part (b) of the lemma. For the second estimate in (b) we argue as follows: 


$$
\begin{aligned}
& \int_{0}^{T} \int_{E(t)} \rho u^{2} r^{m} d r d t \\
& \quad \leqq \int_{0}^{T}\left\|\frac{u}{\theta^{1 / 2}}\right\|_{\infty, R}^{1 / 2}\left(\int_{E(t)} \rho u^{2} r^{m} d r\right)^{3 / 4}\left(\int_{E(t)} \rho \theta r^{m} d r\right)^{1 / 4} d t \\
& \quad \leqq C(T)\left(\int_{0}^{T}\left\|\frac{u}{\theta^{1 / 2}}\right\|_{\infty, R} d t\right)^{1 / 2}\left(\int_{0}^{T} \int_{E(t)} \rho \theta r^{m} d r d t\right)^{1 / 4} \\
& \quad \leqq C(R, T) \omega\left(\int_{0}^{T} \int_{E(t)} \rho r^{m} d r d t ; C(R, T)\right)^{1 / 4}
\end{aligned}
$$

by the bound (22) for the energy, the previous lemma, and the first part of the present argument.

To prove (c) we estimate as follows:

$$
\begin{aligned}
& \int_{0}^{T} \int_{E(t)} \rho(r, t) v(r, t)^{2} r d r d t \\
& \quad \leqq \int_{0}^{T}\left(\int_{E(t)} \rho|v|^{q} r d r\right)^{2 / q}\left(\int_{E(t)} \rho r d r\right)^{(q-2) / q} d t \\
& \quad \leqq C(R)\left(\int_{0}^{T} \int_{E(t)} \rho|r v|^{q} r d r d t\right)^{2 / q}\left(\int_{0}^{T} \int_{E(t)} \rho r d r d t\right)^{(q-2) / q} \\
& \quad \leqq C(R, T)\left(\int_{0}^{T} \int_{E(t)} \rho r d r d t\right)^{(q-2) / q},
\end{aligned}
$$

where we used Lemma 7 in the last step. The argument for $w$ is the same.

\section{Proof of Theorem 1.1}

In this section we complete the proof of Theorem 1.1 by constructing smooth, approximate solutions, applying the a priori bounds of Sections 2 and 3, and taking appropriate limits.

To begin, we let $\left(\rho_{0}, \mathbf{U}_{0}, \theta_{0}\right)$ be initial data satisfying the hypotheses of Theorem 1.1, and we take $\left(\rho_{0}, u_{0}, v_{0}, w_{0}, \theta_{0}\right)=\left(\rho_{0}, U_{0}, \theta_{0}\right)$ to be the corresponding component functions defined for $r \in[0, b]$. We let $H_{\delta}$ be a standard mollifier (in $r$ ) of width $\delta$, and for $a>\delta$ we define smooth, approximate initial data $\left(\rho_{0}^{a, \delta}, U_{0}^{a, \delta}, \theta_{0}{ }^{a, \delta}\right)$ as follows:

- Extend $\rho_{0}$ by its average value outside $[a, b]$, mollify with $H_{\delta}$, restrict to $[a, b]$, and then multiply by a constant to normalize the total mass to be $M_{0}=$ $\int_{0}^{b} \rho_{0} r^{m} d r$. The resulting density function is denoted $\rho_{0}^{a, \delta}(r)$.

- Redefine $w_{0}$ to be its average value and $u_{0}$ and $v_{0}$ to be zero on $[0,2 \delta]$, and redefine all three to be zero on $[b-2 \delta, b]$, then mollify with $H_{\delta}$ to get $u_{0}^{a, \delta}$, $v_{0}^{a, \delta}, w_{0}^{a, \delta}$. Note that these velocities are identically zero on a neighborhood of $r=b$, and $u_{0}^{a, \delta}, v_{0}^{a, \delta}$ and $\left(w_{0}^{a, \delta}\right)_{r}$ are zero on a neighborhood of $r=a$. 
- Redefine $\theta_{0}$ to be its average value on $[0,2 \delta]$ and $[b-2 \delta, b]$, then mollify with $H_{\delta}$ to get $\theta_{0}^{a, \delta}$. Note that $\theta_{0}^{a, \delta}$ is then constant on neighborhoods of $r=a$ and $r=b$.

The resulting data $\left(\rho_{0}^{a, \delta}, U_{0}^{a, \delta}, \theta_{0}^{a, \delta}\right)$ then satisfies the hypotheses (13)-(14) with constants which are independent of $(a, \delta)$. There is then a global-in-time smooth solution $\left(\rho^{a, \delta}, U^{a, \delta}, \theta^{a, \delta}\right)$ of the system (1.4)-(1.8) with boundary conditions as described at the beginning of Section 2 . This is a consequence of the results of FRID \& SHELUKHIN [2] in the spherically symmetric case, and would follow from [2] in the cylindrically symmetric case as well, except that we have imposed the boundary condition $w_{r}=0$ at $a=0$ in place of $w=0$. We instead obtain a global solution for this case as in HoFF [3], by solving first an appropriate system of semidiscrete difference equations, then passing to the limit as the discretization tends to zero. The details are straightforward but rather tedious and technical. Note, however, that $a$ is fixed and positive at this stage of the argument, so that the apparent $1 / r$ singularities in the equations play no role, and the construction of these approximate solutions is essentially a one-space dimensional problem.

For $h, a, \delta>0$ we define the particle paths $r_{h}^{a, \delta}(t)$ associated with this approximate solution by

$$
h=\int_{a}^{r_{h}^{a, \delta}(t)} \rho^{a, \delta}(r, t) r^{m} d r
$$

just as in (24).

\subsection{Convergence of Approximate Solutions}

In the following three propositions we show that there is a subsequence $\left(a_{j}, \delta_{j}\right)$ $\rightarrow(0,0)$ for which the approximate solutions and their associated particle paths converge.

Proposition 1. Let $\left(\rho^{a, \delta}, U^{a, \delta}, \theta^{a, \delta}\right)$ and $r_{h}^{a, \delta}(t)$ be as described above.

(a) There is a subsequence $\left(a_{j}, \delta_{j}\right) \rightarrow(0,0)$ such that $r_{h}^{a_{j}, \delta_{j}}(t)$ converges uniformly for $(h, t)$ in compact subsets of $\left(0, M_{0}\right] \times[0, \infty)$, and the limit $r_{h}(t)$ is Hölder continuous in $(h, t)$ on these compact sets.

(b) If

$$
\underline{r}(t):=\lim _{h \downarrow 0} r_{h}(t)
$$

then

$$
\lim _{t \downarrow 0} \underline{r}(t)=0 .
$$

(c) If the "fluid region" $\mathcal{F}$ is defined by

$$
\mathcal{F}:=\{(r, t): \underline{r}(t)<r \leqq b, 0 \leqq t<\infty,
$$

then $\mathcal{F} \cap\{t>0\} \cap\{r<b\}$ is an open set. 
Proof. We have from the definition (57) of $r_{h}^{a, \delta}$

$$
\frac{\partial r_{h}^{a, \delta}}{\partial t}=u^{a, \delta}, \quad \frac{\partial r_{h}^{a, \delta}}{\partial h}=\frac{n}{\rho^{a, \delta}} .
$$

Uniform Hölder continuity of $\left\{r_{h}^{a, \delta}\right\}$ then follows from the bounds in (26) and Lemma 6 , which show in particular that, for $0 \leqq t \leqq T, h>0$, and $r \geqq r_{h}^{a, \delta}$, $\rho^{a, \delta} \geqq C(h, T)^{-1}$ and $\left|u^{a, \delta}(r, t)\right| \leqq C(h, T) t^{-1 / 4}$. The assertions in (a) then follow from the Ascoli-Arzela theorem.

To prove (b), we take limits in (57) to obtain

$$
h=\int_{0}^{r_{h}(0)} \rho_{0}(r) r^{m} d r \geqq C^{-1} r_{h}(0),
$$

and apply the result of (a) that $r_{h}(\cdot)$ is continuous at $t=0$. The assertion in (c) follows directly from the continuity of the curves $r_{h}(t)$ in $t$ for $h>0$.

Proposition 2. Let the hypotheses and notation of Proposition 1 be in force. Then there is a further subsequence, still denoted by $a_{j}, \delta_{j}$, and limiting functions $U$ and $\theta$ such that

$$
U^{a_{j}, \delta_{j}} \rightarrow U, \quad \theta^{a_{j}, \delta_{j}} \rightarrow \theta
$$

uniformly on compact subsets of $\mathcal{F} \cap\{t>0\}$. The functions $U$ and $\theta$ are Hölder continuous on these compact sets.

Proof. We first prove the uniform Hölder continuity of the approximate temperatures. Thus for $0<\tau \leqq t \leqq T$ and $r_{h_{0}}^{a, \delta}(t) \leqq r_{1} \leqq r_{2} \leqq b$,

$$
\begin{aligned}
\left|\theta^{a, \delta}\left(r_{2}, t\right)-\theta^{a, \delta}\left(r_{1}, t\right)\right| & \leqq \int_{r_{1}}^{r_{2}}\left|\theta_{r}^{a, \delta}(r, t)\right| d r \\
& \leqq \sqrt{r_{2}-r_{1}}\left(\int_{r_{1}}^{r_{2}} \theta_{r}^{a, \delta}(r, t)^{2} d r\right)^{1 / 2} \\
& \leqq C\left(h_{0}, T, \tau\right) \sqrt{r_{2}-r_{1}}
\end{aligned}
$$

by (6); and by (21) and (22),

$$
\int_{r_{h_{0}}^{a, \delta}(t)}^{b} \theta^{a, \delta}(r, t) d r \leqq C\left(h_{0}, T\right) \int_{r_{h_{0}}^{a, \delta}(t)}^{b} \rho^{a, \delta} \theta^{a, \delta} r^{m} d r \leqq C\left(h_{0}, T\right) .
$$

We thus conclude that

$$
\theta^{a, \delta}(r, t) \leqq C\left(h_{0}, \tau, T\right) \quad \text { for } r \geqq r_{h_{0}}^{a, \delta}(t), 0<\tau \leqq t \leqq T,
$$

so that $\left\{\theta^{a, \delta}(r, t)\right\}$ is uniformly bounded and Hölder continuous in $r$ on compact subsets of $\mathcal{F} \cap\{t>0\}$. To prove Hölder continuity in time, we let $0<\tau \leqq t_{1} \leqq$ $t_{2} \leqq T$ and $r_{h_{0}}^{a, \delta}(t)<r_{1}$ for $t \in\left[t_{1}, t_{2}\right]$. Then for $k=\sqrt{t_{2}-t_{1}}$, 


$$
\begin{aligned}
& \left|\theta^{a, \delta}\left(r_{1}, t_{2}\right)-\theta^{a, \delta}\left(r_{1}, t_{1}\right)\right| \\
& \quad \leqq k^{-1} \int_{r_{1}}^{r_{1}+k}\left|\theta^{a, \delta}\left(r, t_{2}\right)-\theta^{a, \delta}\left(r, t_{1}\right)\right| d r+C\left(h_{0}, T, \tau\right) \sqrt{k} \\
& \quad \leqq k^{-1} \int_{r_{1}}^{r_{1}+k} \int_{t_{1}}^{t_{2}}\left|\dot{\theta}^{a, \delta}\right|+\left|u^{a, \delta} \theta_{r}^{a, \delta}\right| d r d t+C\left(h_{0}, T, \tau\right) \sqrt{k} \\
& \quad \leqq \sqrt{\frac{t_{2}-t_{1}}{k}}\left(\int_{r_{1}}^{r_{1}+k} \int_{t_{1}}^{t_{2}}\left|\dot{\theta}^{a, \delta}\right|^{2}+\left|u^{a, \delta} \theta_{r}^{a, \delta}\right|^{2}\right)^{1 / 2}+C\left(h_{0}, T, \tau\right) \sqrt{k} \\
& \quad \leqq C\left(h_{0}, T, \tau\right)\left(\sqrt{\frac{t_{2}-t_{1}}{k}}+\sqrt{k}\right) \\
& \quad \leqq C\left(h_{0}, T, \tau\right)\left|t_{2}-t_{1}\right|^{1 / 4},
\end{aligned}
$$

again by (6). We conclude that $\left\{\theta^{a, \delta}(r, t)\right\}$ is uniformly bounded and Hölder continuous, jointly in $r, t$, on compact subsets of the fluid region $\mathcal{F} \cap\{t>0\}$. A similar argument applies to the velocities $U^{a, \delta}$.

Proposition 3. Assume that the hypotheses and notation of Proposition 2 are in force. Then there is a further subsequence $\left(a_{j}, \delta_{j}\right) \rightarrow(0,0)$ and a function $\rho(r, t)$ such that

$$
\rho^{a_{j}, \delta_{j}}(\cdot, t) \rightarrow \rho(\cdot, t) \quad \text { in } H^{-1}\left([\underline{r}(t)+\eta, b], r^{m} d r\right),
$$

and

$$
\rho^{a_{j}, \delta_{j}}(\cdot, t) \rightarrow \rho(\cdot, t) \quad \text { in } L^{2}\left([\underline{r}(t)+\eta, b], r^{m} d r\right)
$$

for all $t \in[0, T]$ and all $\eta>0$. In addition, if $\rho^{a_{j}, \delta_{j}}(r, t)$ is taken to be zero for $r \leqq a_{j}$, then

$$
\rho^{a_{j}, \delta_{j}}(\cdot, t) \rightarrow 0 \quad \text { in } L^{1}\left([0, \underline{r}(t)], r^{m} d r\right)
$$

when $\underline{r}(t)>0$. Also, for $h>0$ and $T>0$, there is a constant $C=C(h, T)$ such that

$$
C(h, T)^{-1} \leqq \rho \leqq C(h, T)
$$

for $0 \leqq t \leqq T$ and $r_{h}(t) \leqq r \leqq b$. Finally, for $h>0$ and $t \geqq 0$,

$$
h=\int_{\underline{r}(t)}^{r_{h}(t)} \rho(r, t) r^{m} d r .
$$

Proof. Fix a small rectangle

$$
\mathcal{R}=\left[r_{0}, b\right] \times\left[s_{1}, s_{2}\right] \subset\left\{(r, t) \mid r \geqq r_{h}(t) \text { for } t \in[0, T]\right\}
$$

for some $h>0$. Then $C^{-1}(h, T) \leqq \rho^{a}(r, t) \leqq C(h, T)$ for all $(r, t) \in \mathcal{R}$, so that for each time $t \in\left[s_{1}, s_{2}\right]$ the family $\left\{\rho^{a_{j}, \delta_{j}}(\cdot, t)\right\}_{j}$ is bounded in $L^{2}\left(\left[r_{0}, b\right]\right)$, 
uniformly in $j$. Thus if $\left\{t_{k}\right\}$ is a countable, dense set of times, we may pass to a further subsequence, still denoted by $\left(a_{j}, \delta_{j}\right)$, such that

$$
\rho^{a_{j}, \delta_{j}}\left(\cdot, t_{k}\right) \rightarrow \rho\left(\cdot, t_{k}\right) \quad \text { in } L^{2}\left(\left[r_{0}, b\right]\right)
$$

for $t_{k}$ in $\left[s_{1}, s_{2}\right]$. To extend this convergence to all times, we prove strong convergence in $H^{-1}$. Thus define

$$
M^{j}(r, t):=\int_{r_{0}}^{r} \rho^{a_{j}, \delta_{j}}(s, t) d s .
$$

The weak convergence implies that, for each $t_{k}$,

$$
M^{j}\left(r, t_{k}\right) \rightarrow M\left(r, t_{k}\right):=\int_{r_{0}}^{r} \rho\left(s, t_{k}\right) d s
$$

pointwise in $\left[r_{0}, b\right]$, and hence in $L^{2}\left(\left[r_{0}, b\right]\right)$. It follows that if $\psi \in H_{0}^{1}\left(\left[r_{0}, b\right]\right)$ then

$\left|\int_{r_{0}}^{r}\left(\rho^{a_{j}, \delta_{j}}-\rho\right) \psi r^{m} d r\right|=\left|\int_{r_{0}}^{r}\left(M^{j}-M\right) \psi_{\xi} r^{m} d r\right| \leqq C\left\|M^{j}-M\right\|_{L^{2}}\|\psi\|_{H^{1}}$,

so that

$$
\left\|\rho^{a_{j}, \delta_{j}}-\rho\right\|_{H^{-1}} \leqq\left\|M^{j}-M\right\|_{L^{2}} \rightarrow 0
$$

To extend this convergence to all times, we show that the family $\left\{\rho^{a_{j}, \delta_{j}}(\cdot, t)\right\}$ is equicontinuous with respect to $t$ in $H^{-1}\left(\left[r_{0}, b\right]\right)$. Thus for $\tau_{1}, \tau_{2} \in[0, T]$ and $\psi \in H_{0}^{1}\left(\left[r_{0}, b\right]\right)$

$$
\begin{aligned}
& \left|\int_{r_{0}}^{b}\left[\rho^{a_{j}, \delta_{j}}\left(r, \tau_{2}\right)-\rho^{a_{j}, \delta_{j}}\left(r, \tau_{1}\right)\right] \psi r^{m} d r\right| \\
& \quad=\left|\int_{r_{0}}^{b} \int_{\tau_{1}}^{\tau_{2}} \rho^{a_{j}, \delta_{j}} u^{a_{j}, \delta_{j}} \psi \xi r^{m} d r d t\right| \\
& \quad \leqq\|\psi \xi\|_{L^{2}} C(h, T) \int_{\tau_{1}}^{\tau_{2}}\left(\int_{r_{0}}^{b} \rho^{a_{j}, \delta_{j}}\left(u^{a_{j}, \delta_{j}}\right)^{2} r^{m} d r\right)^{1 / 2} d t \\
& \quad \leqq C(h, T)\|\psi\|_{H^{1}}\left|\tau_{2}-\tau_{1}\right| .
\end{aligned}
$$

Therefore

$$
\left\|\rho^{a_{j}, \delta_{j}}\left(\cdot, \tau_{2}\right)-\rho^{a_{j}, \delta_{j}}\left(\cdot, \tau_{1}\right)\right\|_{H^{-1}} \leqq C(h, T)\left|\tau_{2}-\tau_{1}\right| .
$$

It follows that $\rho^{a_{j}, \delta_{j}}(\cdot, t)$ converges strongly in $H^{-1}$ and weakly in $L^{2}$ to $\rho(\cdot, t)$ for all times $t \in\left[s_{1}, s_{2}\right]$. Since $\mathcal{F}$ is a countable union of such rectangles $\mathcal{R}$, there is a further subsequence $\left(a_{j}, \delta_{j}\right) \rightarrow(0,0)$ such that the same convergences hold on $[\underline{r}(t)+\eta, b]$ for each time $t \in[0, T]$ and for all $\eta>0$. 
To prove (59) we fix $t$ and suppose that $\underline{r}(t)>0$. Setting $\rho^{a_{j}, \delta_{j}}=0$ on $\left[0, a_{j}\right]$, we then have, for $a_{j}<r_{h}(t)$,

$$
\begin{aligned}
\int_{0}^{\underline{r}(t)} \rho^{a_{j}, \delta_{j}} r^{m} d r & =\left(\int_{0}^{r_{h}^{a_{j}, \delta_{j}}(t)}+\int_{r_{h}^{a_{j}, \delta_{j}}(t)}^{r_{h}(t)}+\int_{r_{h}(t)}^{\underline{r}^{(t)}}\right) \rho^{a_{j}, \delta_{j}} r^{m} d r \\
& \leqq h+\omega\left(\left|\left[r_{h}^{a_{j}, \delta_{j}}(t), r_{h}(t)\right]\right| ; C_{0}\right)+\omega\left(\left|\left[r_{h}(t), \underline{r}(t)\right]\right| ; C_{0}\right),
\end{aligned}
$$

by Lemma 9. Thus given $\delta>0$, we first choose $h<\delta / 3$ so that the last term is smaller than $\delta / 3$ (which is possible since, by definition, $r_{h}(t) \rightarrow \underline{r}(t)$ ), then $j$ sufficiently large that the middle term is less than $\delta / 3$ (which is possible since, by definition, $\left.r_{h}^{a_{j}, \delta_{j}}(t) \rightarrow r_{h}(t)\right)$. This shows that $\rho^{a_{j}, \delta_{j}}$ tends to zero in $L^{1}([0, \underline{r}(t)])$.

Finally, to prove (60), we fix $t \in[0, T]$ and $h>0$ and choose $l \in(0, h)$. Then taking $\rho^{a_{j}, \delta_{j}}$ to be zero for $r<a_{j}$, we have, for each $j$ (suppressing $t$ ),

$$
\begin{aligned}
h= & \int_{0}^{r_{h}^{a_{j}, \delta_{j}}} \rho^{a_{j}, \delta_{j}} r^{m} d r \\
= & \left(\int_{0}^{\underline{r}}+\int_{\underline{r}}^{r_{l}}+\int_{r_{l}}^{r_{h}}+\int_{r_{h}}^{r_{h}^{a_{j}, \delta_{j}}}\right) \rho^{a_{j}, \delta_{j}} r^{m} d r \\
= & \int_{0}^{\underline{r}} \rho^{a_{j}, \delta_{j}} r^{m} d r+O\left(\omega\left(\left|\left[\underline{r}, r_{l}\right]\right| ; C(h, T)\right)\right) \\
& \quad+\int_{r_{l}}^{r_{h}} \rho^{a_{j}, \delta_{j}} r^{m} d r+O\left(\omega\left(\left|\left[r_{h}, r_{h}^{a_{j}, \delta_{j}}\right]\right| ; C(h, T)\right)\right) .
\end{aligned}
$$

We first let $j \rightarrow \infty$ with $l$ fixed. The first term on the right then goes to zero by the argument above, the last term goes to zero by (1), and the weak $L^{2}$ convergence (58) shows that

$$
\int_{r_{l}}^{r_{h}} \rho^{a_{j}, \delta_{j}} r^{m} d r \rightarrow \int_{r_{l}}^{r_{h}} \rho r^{m} d r
$$

Letting $l \rightarrow 0$ and applying the definition of $\underline{r}$, we then conclude that

$$
h=\int_{\underline{r}(t)}^{r_{h}(t)} \rho r^{m} d r .
$$

Remark. We defined the approximate initial data $\rho_{0}^{a_{j}, \delta_{j}}$ so that for each time $t$,

$$
M_{0}=\int_{0}^{b} \rho_{0} r^{m} d r=\int_{0}^{b} \rho_{0}^{a_{j}, \delta_{j}} r^{m} d r=\int_{0}^{b} \rho^{a_{j}, \delta_{j}}(r, t) r^{m} d r .
$$

It follows that $r_{M_{0}(t)}^{a_{j}, \delta_{j}} \equiv b$ for all $j$ and $t$, so that $r_{M_{0}}(t) \equiv b$. Thus (60) shows in particular that mass is conserved for the limiting solution:

$$
\int_{\underline{r}(t)}^{b} \rho(r, t) r^{m} d r \equiv \int_{0}^{b} \rho_{0}(r) r^{m} d r .
$$




\subsection{Weak forms of the Navier-Stokes equations}

We next turn to the task of proving that the limiting functions are indeed weak solutions of the Navier-Stokes equations in $\Omega \times[0, \infty)$.

First, the limiting functions $\rho, u, v, w$ and $\theta$ have been defined in the fluid region $\mathcal{F}$ but not elsewhere. We therefore define $\rho, \rho u, \rho v, \rho w$ and $\rho \theta$ to be identically zero in the vacuum region $\mathcal{F}^{c}$. As in Section 1 we let $r(x)=|x|$ in the spherically symmetric case and $r(x)=|\tilde{x}|$, where $\tilde{x}=\left(x_{1}, x_{2}, 0\right)$, in the cylindrically symmetric case, so that $\Omega=\{x \mid r(x) \leqq b\}$ in either case. The velocity vector $\mathbf{U}: \bar{\Omega} \times[0, \infty)$ is then defined by

$$
\mathbf{U}(x, t)=u(r(x), t) \frac{x}{r(x)}
$$

in the spherically symmetric case, and by

$$
\begin{aligned}
\mathbf{U}(x, t) & =\mathbf{U}_{\mathrm{rad}}(x, t)+\mathbf{U}_{\tan }(x, t)+\mathbf{U}_{\mathrm{vert}}(x, t) \\
& =u(r(x), t) \frac{\tilde{x}}{r(x)}+v(r(x), t) \frac{\tilde{x}^{\perp}}{r(x)}+w(x, t)(0,0,1)
\end{aligned}
$$

in the cylindrically symmetric case, where $\tilde{x}^{\perp}=\left(-x_{2}, x_{1}, 0\right)$. Abusing notation slightly, we also write $\rho(x, t)$ and $\theta(x, t)$ in place of $\rho(r(x), t)$ and $\theta(r(x), t)$. Similar notation applies to the approximate solutions, for which we now write $\rho^{j}$ in place of $\rho^{a_{j}, \delta_{j}}$, etc.

In the following proposition we show that $(\rho, \mathbf{U}, \theta)$ satisfies the weak form (16) of the mass equation.

Proposition 4. Let $(\rho, \mathbf{U}, \theta)$ be the limit described above in Propositions 1-3.

(a) The weak form (16) of the mass equation holds for $C^{1}$ test functions $\phi: \bar{\Omega} \times$ $\left[t_{1}, t_{2}\right] \rightarrow \mathbf{R}$, which in the cylindrically symmetric case are assumed to vanish for $\left|x_{3}\right| \geqq L$ for some $L$;

(b) $\rho \in C\left([0, \infty) ; W^{1, \infty}(\Omega)^{*}\right)$ in the spherically symmetric case, and $\rho \in$ $C\left([0, \infty) ; W_{L}^{1, \infty}(\Omega)^{*}\right)$ in the cylindrically symmetric case, where

$$
W_{L}^{1, \infty}(\Omega)=\left\{\phi \in W^{1, \infty}(\Omega): \phi \equiv 0 \text { for }\left|x_{3}\right| \geqq L\right\} ;
$$

(c) $\rho^{1 / 2} u \in L^{\infty}\left([0, \infty) ; L^{2}(\Omega)\right)$.

Proof. We first derive the weak form of the one-dimensional equation (6). Thus let $\phi(r, t)$ be a smooth function on $[0, b] \times\left[t_{1}, t_{2}\right]$, so that

$$
\left.\int_{0}^{b} \rho^{j} \phi r^{m} d r\right|_{t_{1}} ^{t_{2}}=\int_{t_{1}}^{t_{2}} \int_{0}^{b}\left(\rho^{j} \phi_{t}+\rho^{j} u^{j} \phi_{r}\right) r^{m} d r d t .
$$

We consider the difference between each of the above terms and the corresponding terms with $\rho^{j}, u^{j}$ replaced by the limits $\rho, u$. First, at time $t=t_{1}$ or $t_{2}$, we have, by Lemma 9 , 


$$
\begin{aligned}
& \left|\int_{0}^{b} \rho^{j} \phi r^{m} d r-\int_{0}^{b} \rho \phi r^{m} d r\right| \\
& \leq\left|\int_{r_{h}}^{b}\left(\rho^{j}-\rho\right) \phi r^{m} d r\right| \\
& \quad+\|\phi\|_{\infty}\left(\int_{0}^{r_{h}^{j}} \rho^{j} r^{m} d r+\int_{r_{h}^{j}}^{r_{h}} \rho^{j} r^{m} d r+\int_{\underline{r}}^{r_{h}} \rho r^{m} d r\right) \\
& \leq\left|\int_{r_{h}}^{b}\left(\rho^{j}-\rho\right) \phi r^{m} d r\right|+\|\phi\|_{\infty}\left(h+\omega\left(\left[r_{h}, r_{h}^{j}\right] ; C_{0}\right)+\omega\left(\left[\underline{r}, r_{h}\right] ; C_{0}\right)\right) .
\end{aligned}
$$

Letting first $j \rightarrow \infty$, then $h \rightarrow 0$, and using the definitions of the curves $r_{h}^{j}, r_{h}$, and $\underline{r}$, together with (58), we obtain

$$
\left.\left.\int_{0}^{b} \rho^{j} \phi r^{m} d r\right|_{t_{1}} ^{t_{2}} \rightarrow \int_{0}^{b} \rho \phi r^{m} d r\right|_{t_{1}} ^{t_{2}}
$$

The same argument applies to the first term on the right-hand side of (61), and for the last term in (61) we have

$$
\begin{aligned}
& \left|\int_{t_{1}}^{t_{2}} \int_{0}^{b} \rho^{j} u^{j} \phi_{r} r^{m} d r d t-\int_{t_{1}}^{t_{2}} \int_{0}^{b} \rho u \phi_{r} r^{m} d r d t\right| \\
& \leqq\|\phi\|_{\infty}\left[\int_{t_{1}}^{t_{2}} \int_{0}^{r_{h}^{j}}\left|\rho^{j} u^{j}\right| r^{m} d r d t+\int_{t_{1}}^{t_{2}} \int_{r_{h}^{j}}^{r_{h}}\left|\rho^{j} u^{j}\right| r^{m} d r d t\right. \\
& \left.\quad+\int_{t_{1}}^{t_{2}} \int_{\underline{r}}^{r_{h}}|\rho u| r^{m} d r d t\right]+\left|\int_{t_{1}}^{t_{2}} \int_{r_{h}}^{b}\left(\rho^{j} u^{j}-\rho u\right) \phi_{r} r^{m} d r d t\right| .
\end{aligned}
$$

For the first term on the right here we have

$$
\begin{aligned}
& \int_{t_{1}}^{t_{2}} \int_{0}^{r_{h}^{j}}\left|\rho^{j} u^{j}\right| r^{m} d r d t \\
& \quad \leqq\left(\int_{t_{1}}^{t_{2}} \int_{0}^{b} \rho^{j} u^{j^{2}} r^{m} d r d t\right)^{1 / 2}\left(\int_{t_{1}}^{t_{2}} \int_{0}^{r_{h}^{j}} \rho^{j} r^{m} d r d t\right)^{1 / 2} \\
& \quad \leqq C(T) \sqrt{h\left(t_{2}-t_{1}\right)} \rightarrow 0 \quad \text { as } h \rightarrow 0
\end{aligned}
$$

The second term on the right in (62) is

$$
\begin{aligned}
& \int_{t_{1}}^{t_{2}} \int_{r_{h}^{j}}^{r_{h}}\left|\rho^{j} u^{j}\right| r^{m} d r d t \\
& \quad \leqq\left(\int_{t_{1}}^{t_{2}} \int_{r_{h}^{j}}^{b} \rho^{j} u^{j^{2}} r^{m} d r d t\right)^{1 / 2}\left(\int_{t_{1}}^{t_{2}} \int_{r_{h}^{j}}^{r_{h}} \rho^{j} r^{m} d r d t\right)^{1 / 2} \\
& \quad \leqq C(T)\left(\int_{t_{1}}^{t_{2}} \omega\left(\left[r_{h}, r_{h}^{j}\right] ; C_{0}\right) d t\right)^{1 / 2}
\end{aligned}
$$


which goes to 0 as $j \rightarrow \infty$ (with $h>0$ fixed) since $r_{h}^{j} \rightarrow r_{h}$ uniformly on $\left[t_{1}, t_{2}\right]$. For the third term on the right-hand side of (62) we have

$$
\int_{t_{1}}^{t_{2}} \int_{\underline{r}}^{r_{h}}|\rho u| r^{m} d r d t \leqq\left(\int_{t_{1}}^{t_{2}} \int_{\underline{r}}^{r_{h}} \rho u^{2} r^{m} d r d t\right)^{1 / 2}\left(\int_{t_{1}}^{t_{2}} \int_{\underline{r}}^{r_{h}} \rho r^{m} d r d t\right)^{1 / 2},
$$

which goes to zero as $h \rightarrow 0$ by Lemma 3.6(a) and by (c) of the present proposition (which is proved independently below). Finally, the last term in (62) goes to zero by the uniform convergence of $u^{j}$ to $u$ and the weak convergence of $\rho^{j}$ to $\rho$. We have thus proved that, for functions $\phi$ which are $C^{1}$ on $[0, b] \times\left[t_{1}, t_{2}\right]$,

$$
\left.\int_{0}^{b} \rho \phi r^{m} d r\right|_{t_{1}} ^{t_{2}}=\int_{t_{1}}^{t_{2}} \int_{0}^{b}\left(\rho \phi_{t}+\rho u \phi_{r}\right) r^{m} d r d t
$$

Now let $\psi: \bar{\Omega} \times\left[t_{1}, t_{2}\right] \rightarrow \mathbf{R}$ be $C^{1}$, and in the spherically symmetric case define

$$
\phi(r, t):=\int_{S} \psi(r y, t) d S_{y},
$$

where the integral is over the unit sphere $S=S^{2}$ in $\mathbf{R}^{2}$; in the cylindrically symmetric case define

$$
\phi\left(r, x_{3}, t\right):=\int_{S} \psi\left(r y, x_{3}, t\right) d S_{y},
$$

where the integral is over the unit circle $S=S^{1}$ in $\mathbf{R}^{2}$. Equation (63) then holds for $\phi$, and it is easy to see that, for $t=t_{1}$ or $t_{2}, \int_{0}^{b} \rho(r, t) \phi(r, t) r^{m} d r$ equals $\int_{\Omega} \rho(x, t) \psi(x, t) d x$ in the spherical case and $\int_{\tilde{\Omega}} \rho(x, t) \psi(x, t) d x_{1} d x_{2}$ in the cylindrical case, where $\tilde{\Omega}=\left\{\left(x_{1}, x_{2}\right) \mid x_{1}^{2}+x_{2}^{2}<b^{2}\right\}$. The first term on the right-hand side of (63) is treated in a similar way, and the second term on the right may be rewritten in the spherically symmetric case as follows:

$$
\begin{aligned}
\int_{t_{1}}^{t_{2}} & \int_{0}^{b} \rho(r, t) u(r, t) \phi_{r}(r, t) r^{m} d r d t \\
& =\int_{t_{1}}^{t_{2}} \int_{0}^{b} \int_{S} \rho(r, t) u(r, t) \nabla \psi(r y, t) \cdot y r^{m} d S_{y} d r d t \\
& =\int_{t_{1}}^{t_{2}} \int_{\Omega} \rho(x, t) u(x, t) \frac{x}{r} \cdot \nabla \psi(x, t) d x d t \\
& =\int_{t_{1}}^{t_{2}} \int_{\Omega} \rho(x, t) \mathbf{U}(x, t) \cdot \nabla \psi(x, t) d x d t .
\end{aligned}
$$

This establishes the weak form of the mass equation in the spherically symmetric case,

$$
\left.\int_{\Omega}(\rho \psi)(x, \cdot) d x\right|_{t_{1}} ^{t_{2}}=\int_{t_{1}}^{t_{2}} \int_{\Omega}\left[\left(\rho \psi_{t}\right)(x, t)+(\rho \mathbf{U} \cdot \nabla \psi)(x, t)\right] d x d t,
$$

for $C^{1}$ functions $\psi: \bar{\Omega} \times\left[t_{1}, t_{2}\right] \rightarrow \mathbf{R}$. 
For the cylindrically symmetric case the above computation shows that for each $x_{3}$ fixed,

$$
\left.\int_{\tilde{\Omega}} \rho \psi d x_{1} d x_{2}\right|_{t_{1}} ^{t_{2}}=\int_{t_{1}}^{t_{2}} \int_{\tilde{\Omega}}\left[\left(\rho \psi_{t}\right)+\mathbf{U}_{\mathrm{rad}} \cdot \nabla \psi\right] d x_{1} d x_{2} d t .
$$

Thus if $\psi\left(x_{1}, x_{2}, x_{3}\right)=0$ for $\left|x_{3}\right| \geqq L$ for some $L$, then

$$
\left.\int_{\Omega} \rho \psi d x\right|_{t_{1}} ^{t_{2}}=\int_{t_{1}}^{t_{2}} \int_{\Omega}\left[\rho \psi_{t}+\mathbf{U}_{\mathrm{rad}} \cdot \nabla \psi\right] d x d t .
$$

Observe that, for such $\psi$,

$$
\int_{t_{1}}^{t_{2}} \int_{\Omega} \rho \mathbf{U}_{\mathrm{vert}} \cdot \nabla \psi d x d t=\int_{t_{1}}^{t_{2}} \int_{\Omega} \rho w\left(\int_{h}^{h} \psi_{x_{3}} d x_{3}\right) r d r d t=0,
$$

and

$$
\begin{aligned}
\int_{t_{1}}^{t_{2}} & \int_{\Omega} \rho \mathbf{U}_{\tan } \cdot \nabla \psi d x d t \\
= & \int_{t_{1}}^{t_{2}} \int_{0}^{b} \rho v\left(\int _ { - L } ^ { L } \int _ { 0 } ^ { 2 \pi } \left[-r \sin (\alpha) \psi_{x}\left(r \cos (\alpha), r \sin (\alpha), x_{3}\right)\right.\right. \\
& \left.\left.+r \cos (\alpha) \psi_{y}\left(r \cos (\alpha), r \sin (\alpha), x_{3}\right)\right] d \alpha d x_{3}\right) d r d t \\
= & \int_{t_{1}}^{t_{2}} \int_{0}^{b} \rho v\left(\int_{-L}^{L} \int_{0}^{2 \pi} \frac{\partial}{\partial \alpha} \psi\left(r \cos (\alpha), r \sin (\alpha), x_{3}\right) d \alpha d x_{3}\right) d r d t=0 .
\end{aligned}
$$

Adding the last three results and recalling that $\mathbf{U}=\mathbf{U}_{\text {rad }}+\mathbf{U}_{\tan }+\mathbf{U}_{\text {vert }}$, we then obtain the weak form of the mass equation in the cylindrically symmetric case,

$$
\left.\int_{\Omega}(\rho \psi)(x, \cdot) d x\right|_{t_{1}} ^{t_{2}}=\int_{t_{1}}^{t_{2}} \int_{\Omega}\left[\left(\rho \psi_{t}\right)(x, t)+(\rho \mathbf{U} \cdot \nabla \psi)(x, t)\right] d x d t,
$$

for $C^{1}$ functions $\psi: \bar{\Omega} \times\left[t_{1}, t_{2}\right] \rightarrow \mathbf{R}$ having compact support in the $x_{3}$ direction. This proves (a).

Proof of (b): First, if $\phi$ is a $C^{1}$ function of $x$, then by the result of (a) for the spherical case,

$$
\begin{aligned}
\left|\int_{\Omega} \rho \phi d x\right|_{t_{1}}^{t_{2}} \mid & =\left|\int_{t_{1}}^{t_{2}} \int_{\Omega} \rho \mathbf{U} \cdot \nabla \phi d x d t\right| \\
& \leqq\|\nabla \phi\|_{\infty} \int_{t_{1}}^{t_{2}}\left(\int_{\Omega} \rho d x\right)^{1 / 2}\left(\int \rho|\mathbf{U}|^{2} d x\right)^{1 / 2} d t \\
& \leqq C(T)\|\nabla \phi\|_{\infty}\left|t_{2}-t_{1}\right| .
\end{aligned}
$$

A straightforward argument enables us to extend this to functions $\phi \in W^{1, \infty}(\Omega)$, so that

$$
\left\|\rho\left(\cdot, t_{2}\right)-\rho\left(\cdot, t_{1}\right)\right\|_{W^{1, \infty}(\Omega)^{*}} \leqq C(T)\left|t_{2}-t_{1}\right|,
$$


for $t_{1}, t_{2} \in[0, T]$. This proves (b) for the spherical case, and the proof for the cylindrical case is similar.

Proof of (c): By (22), there is a constant $C(T)$ such that, for $t \leqq T, \eta>0$, and $j$ sufficiently large,

$$
\int_{\underline{r}(t)+\eta}^{b} \rho^{j}\left|\mathbf{U}^{j}\right|^{2} r^{m} d r d t \leqq C(T) .
$$

We first let $j \rightarrow \infty$, then $\eta \rightarrow 0$ to obtain

$$
\int_{\underline{r}(t)}^{b} \rho|\mathbf{U}|^{2} r^{m} d r d t=\int_{\Omega} \rho|\mathbf{U}|^{2} d x \leqq C(T),
$$

which proves (c).

We now turn to the formulation and proof of the weak form of the momentum equation. Since we cannot determine whether or not a vacuum state $\{\rho=0\}$ occurs at the origin, that is, whether or not $\underline{r} \equiv 0$, we will be able to identify the viscous terms in the momentum equation only as certain limiting distributions. To begin, we define, for given times $t_{1} \leqq t_{2}$ and test functions $\phi$,

$$
\begin{aligned}
\mathcal{U}(j, \phi) & =\mathcal{U}\left(j, \phi, t_{1}, t_{2}\right):=v \int_{t_{1}}^{t_{2}} \int_{a_{j}}^{b} u^{j} \phi_{\xi r} r^{m} d r d t, \\
\mathcal{V}(j, \phi) & =\mathcal{V}\left(j, \phi, t_{1}, t_{2}\right):=\mu \int_{t_{1}}^{t_{2}} \int_{a_{j}}^{b} v^{j} \phi_{\xi r} r^{m} d r d t, \\
\mathcal{W}(j, \phi) & =\mathcal{W}\left(j, \phi, t_{1}, t_{2}\right):=\mu \int_{t_{1}}^{t_{2}} \int_{a_{j}}^{b} w^{j} \phi_{r \xi} r^{m} d r d t .
\end{aligned}
$$

As a first step we treat the simpler case in which $\phi$ (or $\phi_{r}$ in the case of the $w$ equation) vanishes in a neighborhood of the inner boundary.

Lemma 10. Let $\rho, \theta, u, v, w$ be the functions defined above in Propositions 1-3. Let $t_{1}<t_{2}$ and let $\phi$ be a $C^{2}$ function on $[0, b] \times\left[t_{1}, t_{2}\right]$ with $\phi(b, t)=0$ for $t \in\left[t_{1}, t_{2}\right]$.

(a) Assume in addition that $\phi(\cdot, t) \equiv 0$ on $[0, R]$ for some $R>0$ and for all $t \in\left[t_{1}, t_{2}\right]$. Then the limits of $\mathcal{U}(j, \phi)$ and $\mathcal{V}(j, \phi)$ as $j \rightarrow \infty$ exist, and the weak forms of the first two momentum equations (7) and (8) hold in the sense that

$$
\begin{gathered}
\left.\int_{0}^{b} \rho u \phi r^{m} d r\right|_{t_{1}} ^{t_{2}}-\int_{t_{1}}^{t_{2}} \int_{0}^{b}\left(\rho u \phi_{t}+\rho u^{2} \phi_{r}+P \phi_{\xi}+\frac{\rho v^{2} \phi}{r}\right) r^{m} d r d t \\
=\int_{t_{1}}^{t_{2}} \int_{0}^{b} \rho f_{1} \phi r^{m} d r d t+\lim _{j \rightarrow \infty} \mathcal{U}(j, \phi)
\end{gathered}
$$

and

$$
\begin{gathered}
\left.\int_{0}^{b} \rho v \phi r^{m} d r\right|_{t_{1}} ^{t_{2}}-\int_{t_{1}}^{t_{2}} \int_{0}^{b}\left(\rho v \phi_{t}+\rho u v \phi_{r}-\frac{\rho u v \phi}{r}\right) r^{m} d r d t \\
=\int_{t_{1}}^{t_{2}} \int_{0}^{b} \rho f_{2} \phi r^{m} d r d t+\lim _{j \rightarrow \infty} \mathcal{V}(j, \phi) .
\end{gathered}
$$


(b) Assume in addition that $\phi_{r}(\cdot, t) \equiv 0$ on $[0, R]$ for some $R>0$ and for all $t \in\left[t_{1}, t_{2}\right]$ (rather than $\phi(\cdot, t) \equiv 0$ ). Then the limit of $\mathcal{W}(j, \phi)$ as $j \rightarrow \infty$ exists, and the weak form of the third momentum equation (9) holds in the sense that

$$
\begin{gathered}
\left.\int_{0}^{b} \rho w \phi r^{m} d r\right|_{t_{1}} ^{t_{2}}-\int_{t_{1}}^{t_{2}} \int_{0}^{b}\left(\rho w \phi_{t}+\rho u w \phi_{r}\right) r^{m} d r d t \\
=\int_{t_{1}}^{t_{2}} \int_{0}^{b} \rho f_{3} \phi r^{m} d r d t+\lim _{j \rightarrow \infty} \mathcal{W}(j, \phi) .
\end{gathered}
$$

Proof. We outline the proof of (a). If $j$ is large enough that $a_{j}<R$, the weak form of the momentum equation (7) holds for the approximate solutions:

$$
\begin{aligned}
& \left.\int_{0}^{b} \rho^{j} u^{j} \phi r^{m} d r\right|_{t_{1}} ^{t_{2}} \\
& \quad-\int_{t_{1}}^{t_{2}} \int_{0}^{b}\left(\rho^{j} u^{j} \phi_{t}+\rho^{j} u^{j^{2}} \phi_{r}+P^{j} \phi_{\xi}+\frac{\rho^{j} v^{j^{2}} \phi}{r}\right) r^{m} d r d t \\
& \quad=\int_{t_{1}}^{t_{2}} \int_{0}^{b} \rho^{j} f_{1} \phi r^{m} d r d t+\mathcal{U}(j, \phi)
\end{aligned}
$$

(recall that we have extended $\rho^{j}, u^{j}, v^{j}, w^{j}, \theta^{j}$ to be zero outside $\left[a_{j}, b\right]$ ). We proceed by showing that each term in (65) converges to the corresponding term in (64). The convergence of the terms $\rho^{j} u^{j} \phi$ and $\rho^{j} u^{j} \phi_{t}$ is established just as for the term $\rho^{j} u^{j} \phi_{r}$ in the proof of Proposition 4. For the $\rho^{j} u^{j^{2}} \phi_{r}$ term we have

$$
\begin{aligned}
& \left|\int_{t_{1}}^{t_{2}} \int_{0}^{b} \rho^{j} u^{j^{2}} \phi_{r} r^{m} d r d t-\int_{t_{1}}^{t_{2}} \int_{0}^{b} \rho u^{2} \phi_{r} r^{m} d r d t\right| \\
& \quad \leqq \\
& \quad\left\|\phi_{r}\right\|_{\infty}\left(\int_{t_{1}}^{t_{2}} \int_{R}^{r_{h}^{j}(t)} \rho^{j} u^{j^{2}} r^{m} d r d t+\int_{t_{1}}^{t_{2}} \int_{E_{h}^{j}(t)} \rho^{j} u^{j^{2}} r^{m} d r d t\right. \\
& \left.\quad+\int_{t_{1}}^{t_{2}} \int_{E_{h}(t)} \rho u^{2} r^{m} d r d t\right)+\left|\int_{t_{1}}^{t_{2}} \int_{r_{h}(t)}^{b}\left(\rho^{j} u^{j^{2}}-\rho u^{2}\right) \phi_{r} r^{m} d r d t\right|,
\end{aligned}
$$

where $E_{h}^{j}(t)=\left[r_{h}^{j}(t), r_{h}(t)\right] \cap[R, b]\left(\right.$ or $\left.\left[r_{h}(t), r_{h}^{j}(t)\right] \cap[R, b]\right)$ and $E_{h}(t)=$ $\left[\underline{r}(t), r_{h}(t)\right] \cap[R, b]$ (or $\left.\left[r_{h}(t), \underline{r}(t)\right] \cap[R, b]\right)$. We first let $j \rightarrow \infty$ and then $h \rightarrow 0$. The last term in (66) tends to zero because $\rho^{j} \rightarrow \rho$ weakly and $u^{j} \rightarrow u$ strongly in the given region. Next, by Lemma 9 and the definition of the curves $r_{h}^{j}(t)$, the first term on the right-hand side of (66) is bounded by $C(R, T) \omega\left(h\left(t_{2}-t_{1}\right)\right.$; $C(R, T))^{1 / 4}$, which goes to zero as $h \rightarrow 0$. Applying both parts of Lemma 9 , we can bound the second term by 


$$
\begin{aligned}
& \int_{t_{1}}^{t_{2}} \int_{E_{h}^{j}(t)} \rho^{j} u^{j^{2}} r^{m} d r d t \\
& \quad \leqq C(R, T) \omega\left(\int_{t_{1}}^{t_{2}} \int_{E_{h}^{j}(t)} \rho^{j} r^{m} d r d t ; C(R, T)\right)^{1 / 4} \\
& \quad \leqq C(R, T) \omega\left(\int_{t_{1}}^{t_{2}} \omega\left(\left|E_{h}^{j}(t)\right| ; C_{0}\right) d t ; C(R, T)\right)^{1 / 4},
\end{aligned}
$$

which goes to zero as $j \rightarrow \infty$ because $r_{h}^{j}(t) \rightarrow r_{h}(t)$ as $j \rightarrow \infty$ with $h$ fixed. Finally, for the third term on the right-hand side of (66) we can use the fact that $\sqrt{\rho^{j}} u^{j} \rightarrow \sqrt{\rho} u$ in $L^{2}\left([0, b] \times\left[t_{1}, t_{2}\right]\right)$, so that by Lemma 9 again,

$$
\begin{aligned}
& \int_{t_{1}}^{t_{2}} \int_{E_{h}(t)} \rho u^{2} r^{m} d r d t \leqq \liminf _{j \rightarrow \infty} \int_{t_{1}}^{t_{2}} \int_{E_{h}(t)} \rho^{j} u^{j^{2}} r^{m} d r d t \\
& \quad \leqq \liminf _{j \rightarrow \infty} C(R, T) \omega\left(\int_{t_{1}}^{t_{2}} \int_{E_{h}(t)} \rho^{j} r^{m} d r d t ; C(R, T)\right)^{1 / 4} \\
& \quad \leqq C(R, T) \omega\left(\int_{t_{1}}^{t_{2}} \omega\left(\left|E_{h}(t)\right| ; C_{0}\right) d t ; C(R, T)\right)^{1 / 4},
\end{aligned}
$$

which tends to zero as $h \rightarrow 0$. This shows that the $\rho^{j} u^{j^{2}} \phi_{r}$ term in (65) converges to the corresponding term in (64). The other terms in (65) are handled in a similar way.

We now extend the above result to the case that the test function is supported in all of $[0, b]$.

Lemma 11. Fix an increasing $C^{1}$ function $\chi:[0, \infty) \rightarrow[0,1]$ with $\chi \equiv 0$ on $[0,1]$ and $\chi \equiv 1$ on $[2, \infty)$, and define $\chi^{R}(r):=\chi(r / R)$ for $R>0$. Let $\phi$ be a $C^{2}$ function on $[0, b] \times\left[t_{1}, t_{2}\right]$.

(a) Assume that $\phi(0, t)=\phi(b, t)=0$ for $t \in\left[t_{1}, t_{2}\right]$, and define $\phi^{R}:=\chi^{R} \phi$. Then the limits

$$
\lim _{R \rightarrow 0} \lim _{j \rightarrow \infty} \mathcal{U}\left(j, \phi^{R}\right), \quad \lim _{R \rightarrow 0} \lim _{j \rightarrow \infty} \mathcal{V}\left(j, \phi^{R}\right)
$$

exist, and the weak forms of the first two momentum equations (7) and (8) hold in the sense that

$$
\begin{gathered}
\left.\int_{0}^{b} \rho u \phi r^{m} d r\right|_{t_{1}} ^{t_{2}}-\int_{t_{1}}^{t_{2}} \int_{0}^{b}\left(\rho u \phi_{t}+\rho u^{2} \phi_{r}+P \phi_{\xi}+\frac{\rho v^{2} \phi}{r}\right) r^{m} d r d t \\
=\int_{t_{1}}^{t_{2}} \int_{0}^{b} \rho f_{1} \phi r^{m} d r d t+\lim _{R \rightarrow 0} \lim _{j \rightarrow \infty} \mathcal{U}\left(j, \phi^{R}\right)
\end{gathered}
$$

and

$$
\begin{gathered}
\left.\int_{0}^{b} \rho v \phi r^{m} d r\right|_{t_{1}} ^{t_{2}}-\int_{t_{1}}^{t_{2}} \int_{0}^{b}\left(\rho v \phi_{t}+\rho u v \phi_{r}-\frac{\rho u v \phi}{r}\right) r^{m} d r d t \\
=\int_{t_{1}}^{t_{2}} \int_{0}^{b} \rho f_{2} \phi r^{m} d r d t+\lim _{R \rightarrow 0} \lim _{j \rightarrow \infty} \mathcal{V}\left(j, \phi^{R}\right) .
\end{gathered}
$$


(b) Assume now that $\phi_{r}(0, t)=\phi(b, t)=0$ for $t \in\left[t_{1}, t_{2}\right]$, and define

$$
\phi^{R}(r, t):=\phi(0, t)+\chi^{R}(r)[\phi(r, t)-\phi(0, t)] .
$$

Then the limit

$$
\lim _{R \rightarrow 0} \lim _{j \rightarrow \infty} \mathcal{W}\left(j, \phi^{R}\right)
$$

exists, and the weak form of the third momentum equation (9) holds in the sense that

$$
\begin{gathered}
\left.\int_{0}^{b} \rho w \phi r^{m} d r\right|_{t_{1}} ^{t_{2}}-\int_{t_{1}}^{t_{2}} \int_{0}^{b}\left(\rho w \phi_{t}+\rho u w \phi_{r}\right) r^{m} d r d t \\
=\int_{t_{1}}^{t_{2}} \int_{0}^{b} \rho f_{3} \phi r^{m} d r d t+\lim _{R \rightarrow 0} \lim _{j \rightarrow \infty} \mathcal{W}\left(j, \phi^{R}\right) .
\end{gathered}
$$

Proof. We give the proof of (67). The previous lemma applies to the test function $\phi^{R}=\chi^{R} \phi$, so that

$$
\begin{aligned}
& \left.\int_{0}^{b} \rho u \chi^{R} \phi r^{m} d r\right|_{t_{1}} ^{t_{2}} \\
& \quad-\int_{t_{1}}^{t_{2}} \int_{0}^{b}\left(\rho u \chi^{R} \phi_{t}+\rho u^{2}\left(\chi^{R} \phi\right)_{r}+P\left(\chi^{R} \phi\right)_{\xi}+\frac{\rho v^{2} \chi^{R} \phi}{r}\right) r^{m} d r d t \\
& \quad=\int_{t_{1}}^{t_{2}} \int_{0}^{b} \rho f_{1} \chi^{R} \phi r^{m} d r d t+\lim _{j \rightarrow \infty} \mathcal{U}\left(j, \phi^{R}\right) .
\end{aligned}
$$

The first, second, and sixth terms converge to the corresponding terms in (64) as $R \rightarrow 0$ by the Dominated Convergence Theorem. For the third term we have

$$
\int_{t_{1}}^{t_{2}} \int_{0}^{b} \rho u^{2}\left(\chi^{R} \phi\right)_{r}=\int_{t_{1}}^{t_{2}} \int_{0}^{b} \rho u^{2}\left(\chi_{r}^{R} \phi+\chi^{R} \phi_{r}\right) r^{m} d r d t
$$

and the second term on the right here clearly tends to the third term in (64) as $R \rightarrow 0$. Since $\phi(0, t)=0$ we can write $\phi(r, t)=r \psi(r, t)$ for a smooth $\psi$. Then since $\left|\chi_{r}^{R}\right| \leqq C / R$ we can bound the first term on the right-hand side of (71) by

$$
\left|\int_{t_{1}}^{t_{2}} \int_{R}^{2 R} \rho u^{2} C \frac{r}{R} \psi(r) r^{m} d r d t\right| \leqq C \int_{t_{1}}^{t_{2}} \int_{R}^{2 R} \rho u^{2} r^{m} d r d t,
$$

which tends to zero as $R \rightarrow 0$ by the bound on the limiting energy. The same argument applies to the the fourth and fifth terms in (70). This proves the result for the $u$ equation. The proofs of (68) and (69) are essentially the same.

We can now state the momentum equation for the spherically symmetric case.

Proposition 5. The weak form (17) of the momentum equation holds for the spherically symmetric case, as stated in Theorem 1.1(d). 
Proof. Given $\psi$ as described in the theorem, define

$$
\phi(r, t):=\int_{S^{2}} \psi(r y, t) y_{i} d S_{y},
$$

for fixed $i=1,2,3$. Then $\phi(0, t)=\phi(b, t) \equiv 0$ so that by Lemma 11 ,

$$
\begin{gathered}
\left.\int_{0}^{b} \rho u \phi r^{m} d r\right|_{t_{1}} ^{t_{2}}-\int_{t_{1}}^{t_{2}} \int_{0}^{b}\left(\rho u \phi_{t}+\rho u^{2} \phi_{r}+P \phi_{\xi}\right) r^{m} d r d t \\
=\int_{t_{1}}^{t_{2}} \int_{0}^{b} \rho f_{1} \phi r^{m} d r d t+\lim _{R \rightarrow 0} \lim _{j \rightarrow \infty} \mathcal{U}\left(j, \phi^{R}\right) .
\end{gathered}
$$

We convert each of these terms to integrals in Cartesian coordinates involving $\psi$. The derivations are very much like those occurring in the proof of Proposition 4, except for the last term, which we look at in some detail. Recalling the definitions in Lemma 11, we have

$$
\phi^{R}(r, t)=\int_{S^{2}} \psi^{R}(r y, t) y_{i} d S_{y},
$$

where $\psi^{R}(x, t):=\chi^{R}(|x|) \psi(x, t)$. Then since $\phi^{R}=0$ at $r=a_{j}, b$,

$$
\begin{aligned}
\int_{a_{j}}^{b} u^{j} \phi_{\xi r}^{R} r^{m} d r & =-\int_{a_{j}}^{b} u_{\xi}^{j} \phi_{\xi}^{R} r^{m} d r \\
& =-\int_{a_{j}}^{b}\left(u_{r}^{j}+\frac{m u^{j}}{r}\right)\left(\phi_{r}^{R}+\frac{m \phi^{R}}{r}\right) r^{m} d r \\
& =-\int_{a_{j}}^{b}\left[u_{r}^{j} \phi_{r}^{R}+\frac{m}{r}\left(u^{j} \phi^{R}\right)_{r}+\frac{m^{2} u^{j} \phi^{R}}{r^{2}}\right] r^{m} d r \\
& =-\int_{a_{j}}^{b}\left(u_{r}^{j} \phi_{r}^{R}+\frac{m u^{j} \phi^{R}}{r^{2}}\right) r^{m} d r \\
& =-\int_{a_{j}}^{b}\left[r\left(\frac{u^{j}}{r}\right)_{r} \phi_{r}^{R} r^{m}+\frac{u^{j}}{r}\left(r^{m} \phi^{R}\right)_{r}\right] d r .
\end{aligned}
$$

Summing over repeated indexes, we then have

$$
\begin{aligned}
\int_{a_{j}}^{b} u^{j} \phi_{\xi r}^{R} r^{m} d r= & -\int_{a_{j}}^{b} r\left(\frac{u^{j}}{r}\right)_{r}\left(\int_{S^{2}} \psi_{x_{k}}^{R}(r y, t) y_{k} y_{i} d S_{y}\right) r^{m} d r \\
& -\int_{a_{j}}^{b} \frac{u^{j}}{r}\left(\int_{S^{2}} \psi_{x_{i}}^{R}(r y, t) d S_{y}\right) r^{m} d r
\end{aligned}
$$




$$
\begin{aligned}
& =-\int_{a_{j}}^{b} \int_{S^{2}}\left[r\left(\frac{u^{j}}{r}\right)_{r} y_{k} y_{i}+\frac{u^{j}}{r} \delta_{i k}\right] \psi_{y_{k}}^{R}(r y, t) r^{m} d S_{y} d r \\
& =-\int_{\Omega}\left[\left(\frac{u^{j}(|x|, t)}{|x|}\right)_{r} \frac{x_{k} x_{i}}{|x|}+\frac{u^{j}}{|x|} \delta_{i k}\right] \psi_{x_{k}}^{R}(x, t) d x \\
& =-\int_{\Omega}\left(u^{j}(|x|, t) \frac{x_{i}}{|x|}\right)_{x_{k}} \psi_{y_{k}}^{R}(x, t) d x \\
& =\int_{\Omega} u^{j}(|x|, t) \frac{x_{i}}{|x|} \Delta\left(\psi^{R}\right) d x .
\end{aligned}
$$

Thus

$$
\mathcal{U}\left(j, \phi^{R}\right)=v \int_{t_{1}}^{t_{2}} \int_{\Omega} u^{j}(|x|, t) \frac{x_{i}}{|x|} \Delta\left(\psi^{R}\right) d x d t .
$$

The result then follows because

$$
\int_{\Omega} u^{j}(|x|, t) \frac{x_{i}}{|x|} \Delta\left(\psi^{R}\right) d x=\int_{\Omega} \mathbf{U}^{j} \cdot \nabla \psi_{x_{i}}^{R} d x
$$

and $v=\lambda+2 \mu$.

In the following proposition we derive the weak form of the momentum equation for the cylindrically symmetric case.

Proposition 6. The weak form (17) of the momentum equation holds for the cylindrically symmetric case, as stated in Theorem 1.1(d).

Proof. Given $\psi$ as described in the statement of the theorem, define the test function

$$
\phi_{i}(r, t):=\int_{-L}^{L} \int_{S^{1}} \psi\left(r y, x_{3}, t\right) y_{i} d S_{y} d x_{3}
$$

for $i=1,2$. Then $\phi_{i}\left(0, x_{3}, t\right)=\phi_{i}\left(b, x_{3}, t\right)=0$, so that part (a) of Lemma 11 applies to give

$$
\begin{aligned}
& \left.\int_{0}^{b} \rho u \phi_{i} r d r\right|_{t_{1}} ^{t_{2}}-\int_{t_{1}}^{t_{2}} \int_{0}^{b}\left(\rho u \phi_{i, t}+\rho u^{2} \phi_{i, r}+P \phi_{i, \xi}+\frac{\rho v^{2} \phi_{i}}{r}\right) r d r d t \\
& =\int_{t_{1}}^{t_{2}} \int_{0}^{b} \rho f_{1} \phi_{i} r d r d t+\lim _{R \rightarrow 0} \lim _{j \rightarrow \infty} \mathcal{U}\left(j, \phi_{i}^{R}\right)
\end{aligned}
$$

and

$$
\begin{gathered}
\left.\int_{0}^{b} \rho v \phi_{i} r d r\right|_{t_{1}} ^{t_{2}}-\int_{t_{1}}^{t_{2}} \int_{0}^{b}\left(\rho v \phi_{i, t}+\rho u v \phi_{i, r}-\frac{\rho u v \phi_{i}}{r}\right) r d r d t \\
=\int_{t_{1}}^{t_{2}} \int_{0}^{b} \rho f_{2} \phi_{i} r d r d t+\lim _{R \rightarrow 0} \lim _{j \rightarrow \infty} \mathcal{V}\left(j, \phi_{i}^{R}\right)
\end{gathered}
$$


where

$$
\phi_{i}^{R}\left(r, x_{3}, t\right)=\int_{-h}^{h} \int_{S^{1}} \psi^{R}\left(r y, x_{3}, t\right) y_{i} d S_{y} d x_{3} \quad \text { for } i=1,2 .
$$

We subtract (74) with $i=2$ from (73) with $i=1$ to obtain

$$
\begin{aligned}
\int_{0}^{b} & \left.\rho\left(u \phi_{1}-v \phi_{2}\right) r d r\right|_{t_{1}} ^{t_{2}}-\int_{t_{1}}^{t_{2}} \int_{0}^{b}\left[\rho\left(u \phi_{1, t}-v \phi_{2, t}\right)+\left(\rho u^{2} \phi_{1, r}-\rho u v \phi_{2, r}\right)\right. \\
& \left.+P \phi_{1, \xi}+\left(\frac{\rho v^{2} \phi_{1}}{r}+\frac{\rho u v \phi_{2}}{r}\right)\right] r d r d t \\
= & \int_{t_{1}}^{t_{2}} \int_{0}^{b} \rho\left(f_{1} \phi_{1}-f_{2} \phi_{2}\right) r d r d t+\lim _{R \rightarrow 0} \lim _{j \rightarrow \infty}\left[\mathcal{U}\left(j, \phi_{1}^{R}\right)-\mathcal{V}\left(j, \phi_{2}^{R}\right)\right] \cdot(75)
\end{aligned}
$$

We compute the first term on the left as follows:

$$
\begin{aligned}
\int_{0}^{b} \rho\left(u \phi_{1}-v \phi_{2}\right) r d r & =\int_{-L}^{L} \int_{S^{1}} \int_{0}^{b} \rho\left(u y_{1}-v y_{2}\right) \psi\left(r y, x_{3}, t\right) r d r d S_{y} d x_{3} \\
& =\int_{\Omega} \rho \mathbf{U}^{1} \psi d x .
\end{aligned}
$$

The argument is similar for the other terms in (75) except for the last term on the right, which is handled as in Proposition 6. This proves (17) for the $i=1$ component, and the proof for $i=2$ is similar.

For the case $i=3$ we define the test function

$$
\phi\left(r, x_{3}, t\right):=\int_{S^{1}} \psi\left(r y, x_{3}, t\right) d S_{y} .
$$

Then $\phi\left(b, x_{3}, t\right)=\phi_{r}\left(0, x_{3}, t\right)=0$, and part (b) of Lemma 11 applies. Integrating (69) with respect to $x_{3}$ over $[-L, L]$ we thus obtain

$$
\begin{gathered}
\left.\int_{-L}^{L} \int_{0}^{b} \rho w \phi r d r d x_{3}\right|_{t_{1}} ^{t_{2}}-\int_{t_{1}}^{t_{2}} \int_{-L}^{L} \int_{0}^{b}\left(\rho w \phi_{t}+\rho u w \phi_{r}\right) r d r d x_{3} d t \\
=\int_{t_{1}}^{t_{2}} \int_{-L}^{L} \int_{0}^{b} \rho f_{3} \phi r d r d x_{3} d t+\lim _{R \rightarrow 0} \lim _{j \rightarrow \infty} \int_{-L}^{L} \mathcal{W}\left(j, \phi^{R}\left(\cdot, x_{3}, \cdot\right)\right) d x_{3} .
\end{gathered}
$$

For the first term on the left-hand side we have (at either $t=t_{1}$ or $t=t_{2}$ )

$$
\begin{aligned}
\int_{-L}^{L} \int_{0}^{b} \rho w \phi r d r d x_{3} & =\int_{-L}^{L} \int_{0}^{b} \int_{S^{1}}\left(\rho \mathbf{U}^{3}\right)(r y, t) \psi\left(r y, x_{3}, t\right) r d S_{y} d r d x_{3} \\
& =\int_{\Omega} \rho \mathbf{U}^{3} \psi d x .
\end{aligned}
$$

Similarly,

$$
\int_{t_{1}}^{t_{2}} \int_{-L}^{L} \int_{0}^{b} \rho w \phi_{t} r d r d x_{3} d t=\int_{t_{1}}^{t_{2}} \int_{\Omega} \rho \mathbf{U}^{3} \psi_{t} d x d t
$$


and

$$
\int_{t_{1}}^{t_{2}} \int_{-L}^{L} \int_{0}^{b} \rho f_{3} \phi r d r d x_{3} d t=\int_{t_{1}}^{t_{2}} \int_{\Omega} \rho \mathbf{F}^{3} \psi d x d t
$$

Next we observe that

$$
\begin{aligned}
\int_{t_{1}}^{t_{2}} & \int_{\Omega} \rho \mathbf{U}^{3} \mathbf{U} \cdot \nabla \psi d x d t \\
= & \int_{t_{1}}^{t_{2}} \int_{-L}^{L} \int_{0}^{b} \int_{S^{1}}(\rho w)(r y, t)\left[\left(u x_{1}-v x_{2}\right) \psi_{x_{1}}\right. \\
& \left.+\left(u x_{2}+v x_{1}\right) \psi_{x_{2}}\right] d S_{y} d r d x_{3} d t \\
& +\int_{t_{1}}^{t_{2}} \int_{0}^{b} \int_{S^{1}} w(r y, t)\left(\int_{-L}^{L} \psi_{x_{3}} d x_{3}\right) r d S_{y} d r d t \\
= & \int_{t_{1}}^{t_{2}} \int_{-L}^{L} \int_{0}^{b} \int_{S^{1}}(\rho u w)(r, t) \nabla_{\tilde{x}} \psi\left(r y, x_{3}, t\right) \cdot y r d S_{y} d r d x_{3} d t \\
& +\int_{t_{1}}^{t_{2}} \int_{-L}^{L} \int_{0}^{b}(\rho v w)(r, t)\left(\int_{S^{1}}-y_{2} \psi_{x_{1}}+y_{1} \psi_{x_{2}} d S_{y}\right) d r d x_{3} d t \\
= & \int_{t_{1}}^{t_{2}} \int_{-L}^{L} \int_{0}^{b} \rho u w \phi_{r} r d r d x_{3} d t .
\end{aligned}
$$

We have used here the facts that

$$
\int_{-h}^{h} \psi_{x_{3}} d x_{3}=0, \quad \text { and } \quad \int_{S^{1}}\left(-y_{2} \psi_{x_{1}}+y_{1} \psi_{x_{2}}\right) d S_{y}=\int_{S^{1}} \frac{d \psi}{d \alpha} d \alpha=0 .
$$

This takes care of the last term on the left-hand side of (76) and the computation of the $\mathfrak{U}_{3}$ term is carried out as in Proposition 6.

Concerning the weak form of the energy equation, it is straightforward to prove (18) for test functions supported in the fluid region, as asserted in (e) of Theorem 1.1. The proof consists in applying the strong convergence of the approximate velocities and temperatures and the weak convergence of the approximate densities, and converting to Cartesian coordinates as in the previous two propositions. We omit the details, but we note that the assertion in (e) that $\nabla \mathbf{U}, \nabla \theta \in$ $L_{\text {loc }}^{1}(\mathcal{F})$ is a consequence of $(23),(26)$, and (4).

Proposition 7. The weak form (18) of the energy equation holds as stated in Theorem 1.1(e).

Finally, we prove a result concerning the balance of total energy.

Proposition 8. The total energy $\mathcal{E}$ of the limiting solution satisfies the balance relation described in Theorem 1.1(f). 
Proof. We give the proof for the spherically symmetric case, the proof for the cylindrically symmetric being nearly identical. Denoting the energy associated with the approximate solution $\left(\rho^{j}, \mathbf{U}^{j}, \theta^{j}\right)$ by

$$
\mathcal{E}^{j}=\int_{0}^{T} \int_{\Omega} \rho^{j}(x, t)\left[\frac{1}{2}\left|\mathbf{U}^{j}(x, t)\right|^{2}+\theta^{j}(x, t)\right] d x d t,
$$

we have, from the energy equation (3),

$$
\mathcal{E}^{j}(t)=\mathcal{E}^{j}(0)+\int_{0}^{t} \int_{a_{j} \leqq r(x) \leqq b} \rho^{j} \mathbf{U}^{j} \cdot \mathbf{F} d x d s .
$$

Now let $\phi \in \mathcal{D}(0, \infty)$ with support in $(0, T)$. Then for $R \geqq a_{j}>0$,

$$
\begin{aligned}
\mathcal{E}^{j}(0) & \int_{0}^{T} \phi(t) d t \\
= & \int_{0}^{T}\left[\mathcal{E}^{j}(t)-\int_{0}^{t} \int_{a_{j} \leqq r(x) \leqq b} \rho^{j} \mathbf{U}^{j} \cdot \mathbf{F} d x d s\right] \phi(t) d t \\
= & \int_{0}^{T}\left(\int_{a_{j}}^{R}+\int_{R}^{R \vee \underline{r}(t)}+\int_{R \vee \underline{r}(t)}^{R \vee r_{h}(t)}+\int_{R \vee r_{h}(t)}^{b}\right)\left(\rho^{j} E^{j}\right)(r, t) \phi(t) r^{2} d r d t \\
& -\int_{0}^{T} \int_{0}^{t} \int_{a_{j} \leqq r(x) \leqq b} \rho^{j} \mathbf{U}^{j} \cdot \mathbf{F} d x d s \phi(t) d t,
\end{aligned}
$$

where $E^{j}=\theta^{j}+\frac{1}{2}\left|\mathbf{U}^{j}\right|^{2}$. We first let $j \rightarrow \infty$, then $h \rightarrow 0$, then finally $R \rightarrow 0$. It is clear that $\mathcal{E}^{j}(0) \rightarrow \mathcal{E}(0)$ as $j \rightarrow \infty$, and

$$
\int_{0}^{T} \int_{0}^{t} \int_{a_{j} \leqq r(x) \leqq b} \rho^{j} \mathbf{U}^{j} \cdot \mathbf{F} d x d s \phi(t) d t \rightarrow \int_{0}^{T} \int_{0}^{t} \int_{\Omega} \rho \mathbf{U} \cdot \mathbf{F} d x d s \phi(t) d t
$$

by the same argument used to prove the convergence of the $\rho^{j} \mathbf{U}^{j} \phi_{r}$ term in (61). For the second-last term on the right-hand side of (77) we apply the strong convergence of $E^{j}$ and the weak convergence of $\rho^{j}$ in the given region to obtain

$$
\begin{aligned}
\int_{0}^{T} \int_{R \vee r_{h}(t)}^{b} \phi \rho^{j} E^{j} r^{2} d r d t & \rightarrow \int_{0}^{T} \int_{R \vee r_{h}(t)}^{b} \phi \rho E r^{2} d r d t \quad \text { as } j \rightarrow \infty \\
& \rightarrow \int_{0}^{T} \int_{R \vee \underline{r}(t)}^{b} \phi \rho E r^{2} d r d t \quad \text { as } h \rightarrow 0 \\
& \rightarrow \int_{0}^{T} \int_{\underline{r}(t)}^{b} \phi \rho E r^{2} d r d t \quad \text { as } R \rightarrow 0,
\end{aligned}
$$

where we have used the Dominated Convergence Theorem and the integrability of the total energy in the two last limits. For the second integral on the right-hand side of (77) we apply Lemma 9: 


$$
\begin{aligned}
& \int_{0}^{T} \int_{R}^{R \vee \underline{r}(t)} \phi \rho^{j} E^{j} r^{2} d r d t \\
& \leqq\|\phi\|_{\infty} C(R, T)\left[\omega\left(\int_{0}^{T} \int_{R}^{R \vee \underline{r}(t)} \rho^{j} r^{2} d r d t ; C(R, T)\right)\right. \\
& \left.\quad+\omega\left(\int_{0}^{T} \int_{R}^{R \vee \underline{r}(t)} \rho^{j} r^{2} d r d t ; C(R, T)\right)^{1 / 4}\right]
\end{aligned}
$$

which tends to zero as $j \rightarrow \infty$, for fixed $R$, by (59). For the the third integral in (77) we apply the first two parts of Lemma 9 to get

$$
\begin{aligned}
& \int_{0}^{T} \int_{R \vee \underline{r}(t)}^{R \vee r_{h}(t)} \phi \rho^{j} E^{j} r^{2} d r d t \\
& \leq\|\phi\|_{\infty} C(R, T)\left[\omega\left(\int_{0}^{T} \omega\left(\left|\left[R \vee \underline{r}(t), R \vee r_{h}(t)\right]\right| ; C_{0}\right) d t ; C(R, T)\right)\right. \\
& \left.\quad+\omega\left(\int_{0}^{T} \omega\left(\left|\left[R \vee \underline{r}(t), R \vee r_{h}(t)\right]\right| ; C_{0}\right) d t ; C(R, T)\right)^{1 / 4}\right],
\end{aligned}
$$

which tends to zero as $h \rightarrow 0$, for fixed $R$, by definition of $\underline{r}(t)$. We thus conclude from (77) that

$$
\begin{aligned}
\int_{0}^{T} \mathcal{E}(0) \phi(t) d t= & \lim _{R \rightarrow 0} \lim _{j \rightarrow \infty} \int_{0}^{T}\left(\int_{a_{j}}^{R} \rho^{j} E^{j} r^{2} d r\right) \phi(t) d t \\
& +\int_{0}^{T}\left(\int_{\underline{r}(t)}^{b} \rho E r^{2} d r\right) \phi(t) d t \\
& -\int_{0}^{T}\left(\int_{0}^{t} \int_{\Omega} \rho \mathbf{U} \cdot \mathbf{F} d x d s\right) \phi(t) d t
\end{aligned}
$$

as required.

\subsection{Proof of Theorem 1.1}

Part (a) of Theorem 1.1 is Proposition 1, the semicontinuity being implicit in the proof. The existence and regularity of $\rho, \mathbf{U}, \theta$ asserted in (b) of Theorem 1.1 follow from Propositions 2 and 3. The weak forms of the mass and momentum equations are proved in Propositions 4, 5, and 6, from which the regularity assertions in (c) and (d) of Theorem 1.1 follow immediately. Finally, the results in (e) and (f) of the Theorem 1.1 are proved in Propositions 7 and 8.

Acknowledgements. Research supported in part by the NSF under Grants DMS-9986658 and DMS-0305072 (HOFF) and Grant DMS-0206631 (JENSSEN). 


\title{
References
}

1. FEIREISL, E.: The dynamical system approach to the Navier-Stokes equations of compressible fluids. Advances in Math. Fluid Mech. (Paseky, 1999), Springer, Berlin, 2000, pp. 35-66

2. FRID, H., SHELUKHIN, V.: Vanishing shear viscosity in the equations of compressible fluids for the flows with the cylinder symmetry. SIAM J. Math. Anal. 31, 1144-1156 (2000)

3. HofF, D.: Spherically symmetric solutions of the Navier-Stokes equations for compressible, isothermal flow with large, discontinuous initial data. Indiana Univ. Math. J. 41, 1225-1302 (1992)

4. HofF, D.: Discontinuous solutions of the Navier-Stokes equations for multidimensional flows of heat-conducting fluids. Arch. Rational Mech. Anal. 139, 303-354 (1997)

5. KAZHIKHOV, A.V., SHELUKHIN V.V.: Unique global solution with respect to time of initial-boundary value problems for one-dimensional equations of a viscous gas. J. Appl. Math. Mech. 41, 273-282 (1977); translated from Prikl. Mat. Meh. 41, 282-291 (1977) (Russian)

6. LIONS, P.L.: Mathematical topics in Fluid Mechanics. Vol. 2, Oxford University Press, Oxford, 1998

\author{
Department of Mathematics \\ Indiana University \\ Bloomington, IN 47405 \\ and \\ Department of Mathematics \\ North Carolina State University \\ Raleigh, NC 27695
}

(Accepted February 6, 2004)

Published online June 7, 2004 - (C) Springer-Verlag (2004) 